\title{
Reconsidering Power Functional Theory
}

\author{
James F. Lutskd* \\ Center for Nonlinear Phenomena and Complex Systems CP 231, \\ Université Libre de Bruxelles, Blvd. du Triomphe, 1050 Brussels, Belgium \\ Martin Oettel \\ Institute for Applied Physics, University of Tübingen, \\ Auf der Morgenstelle 10, 72076 Tübingen, Germany
}

(Dated: August 11, 2021)

\begin{abstract}
The original derivation of Power Functional Theory, Schmidt and Brader, JCP138, 214101 (2013), is reworked in some detail with a view to clarifying and simplifying the logic and making explicit the various functional dependencies. We note various issues with the original development and suggest a modification that allows us to avoid them. In the process we also suggest an alternative interpretation of our results that bears surprising similarities to classical Density Functional Theory.
\end{abstract}

\footnotetext{
* http://www.lutsko.com; jlutsko@ulb.ac.be

† martin.oettel@uni-tuebingen.de
} 


\section{INTRODUCTION}

Classical Density Functional Theory (cDFT) [1, 2] has proven to be a powerful tool in the study of inhomogeneous classical systems. In his seminal review article that helped to define the field, Evans also discussed a dynamic extension that has come to be known as Dynamical Density Functional Theory (DDFT) which is applicable to particles obeying an over-damped Brownian dynamics. The two are intimately related since the free energy functionals that play a central role in $\mathrm{cDFT}$ are used in DDFT to describe the deterministic driving force governing the evolution of the local density [3- 5$]$. While cDFT is based on mathematically exact theorems - and as such, represents a formally equivalent reformulation of some aspects of classical statistical mechanics - DDFT is heuristic, depending in all of its various derivations on a kind of uncontrolled, local-equilibrium approximation. It would obviously be desirable if the paradigm of DFT could be extended to non-equilibrium systems thus giving a formally equivalent theory that, like cDFT, admits of simple but highly useful approximations.

This is precisely the goal of Power Functional Theory (PFT) as first proposed by Schmidt and Brader[6] (hereafter referred to as SB) for particles subject to an over-damped Brownian dynamics and subsequently extended by Schmidt and co-workers over the last several years to quantum[7], Newtonian[8] and active-particle[9] systems. The basic idea of the various developments is to introduce a functional the minimization of which generates the time-dependent many-particle distribution function for the system. A version of constrained search (described below) is used to project this onto a functional that depends only on the local density (as in cDFT) and the local current, where the latter is the key to accessing time-dependent quantities. Minimization with respect to these fields yields the actual timedependent density and current. A significant development inspired by this work, but not directly dependent on it, has been the study of so-called "non-adiabatic" dynamics - basically, the difference between some non-equilibrium dynamics followed via simulation and the predictions of DDFT[10, 11]. This has provided insight into the limits of DDFT (which itself has become increasingly popular, see e.g. the recent review of te Vrugt, Löwen and Wittkowski[12]) as well as some heuristic approaches to modeling these differences. Recently,

PFT has also been used to motivate models of phase coexistence in active particles $[13-15]$.

In the following, we review the development of PFT using a notation that is constructed to 
make particularly evident the functional dependencies which can get lost with more standard, and less detailed, notation. The necessary calculations are presented in a fairly explicit manner to avoid ambiguities as good as possible. In doing so, certain inconsistencies will be noted that, taken together, throw doubt on the validity of the framework. A modification aimed at overcoming these difficulties is proposed and yields new insights into the possibility of fulfilling the program proposed by SB. Section II of the paper sets the stage with a brief review of Brownian dynamics and also introduces the functional notation. Section III summarizes the key points of the exact PFT formalism including the generating functional for the time-dependent $N$-body distribution function, its projection onto the density-current subspace and some of the structural elements stressed by SB and, in the processes, various problems in the derivation are highlighted. In Section IV we present our modification of the original PFT of SB that allows us to complete their program with surprising thoroughness. In the process, we note an alternative interpretation of our results which may provide a conceptual reformulation of Brownian Dynamics that is closer to the goals of SB than was our original development. The paper ends with a brief summary of our results.

\section{BROWNIAN DYNAMICS}

The starting point is a system of $N$ identical, classical particles of mass $m=1$ in $D$ dimensions for which the $i$-th particle has coordinates $\mathbf{q}^{(i)}$ and velocities $\mathbf{v}^{(i)}$. The particles interact via a conservative potential $U\left(\mathbf{q}^{(1)}, \ldots, \mathbf{q}^{(N)}\right)$ which will be written more briefly as $U\left(\mathbf{q}^{N}\right)$ where $\mathbf{q}^{N}$ is the collection of all $N$ coordinates. In the following, a slightly compressed notation will be used whereby the position (and time) arguments are written as subscripts so that the potential will be written as $U_{\mathbf{q}^{N}}$. The particles also experience a (possibly) timedependent external one-body potential $\phi_{t \mathbf{r}}$. Again, what is written here as $\phi_{t \mathbf{r}}$ would, more conventionally be written as $\phi(\mathbf{r}, t)$, and in the following, the time argument will always precede the position argument(s) in the subscript notation. Finally, there is a stochastic force and corresponding friction proportional to the velocity (representing, e.g., a bath of 
much smaller particles) so that the equations of motion are

$$
\begin{aligned}
\frac{d}{d t} \widehat{\mathbf{q}}_{t}^{(i)} & =\widehat{\mathbf{v}}_{t}^{(i)} \\
\frac{d}{d t} \widehat{\mathbf{v}}_{t}^{(i)} & =-\gamma \widehat{\mathbf{v}}_{t}^{(i)}-\nabla_{i} U_{\widehat{\mathbf{q}}_{t}^{N}}-\sum_{i=1}^{N} \nabla_{i} \phi_{t \widehat{\mathbf{q}}_{t}^{(i)}}+\widehat{\boldsymbol{\xi}}_{t}^{(i)}
\end{aligned}
$$

where a caret (hat) indicates a stochastic variable, $\gamma$ is the friction and $\widehat{\boldsymbol{\xi}}_{t}^{(i)}$ are $D$ dimensional vectors whose components are Gaussian-distributed white noise with correlations $\left\langle\widehat{\boldsymbol{\xi}}_{t}^{(i)} \widehat{\boldsymbol{\xi}}_{t^{\prime}}^{(j)}\right\rangle=2 \gamma k_{B} T \delta_{i j} \delta\left(t-t^{\prime}\right) \mathbb{1}$ (where $\mathbb{1}$ is the unit tensor for the Cartesian components). Following DDFT, the original PFT was developed for the over-damped limit in which the time-derivative of the velocity can be neglected (this can be justified rigorously with certain scaling assumptions, see e.g. Goddard, et al.[16]) giving the so-called Brownian dynamics

$$
\frac{d}{d t} \widehat{\mathbf{q}}_{t}^{(i)}=\widehat{\mathbf{v}}_{t}^{(i)}=-\frac{1}{\gamma} \nabla_{i} U_{\widehat{\mathbf{q}}_{t}^{N}}-\frac{1}{\gamma} \sum_{i=1}^{N} \nabla_{i} \phi_{t \widehat{\mathbf{q}}_{t}^{(i)}}+\frac{1}{\gamma} \widehat{\boldsymbol{\xi}}_{t}^{(i)} .
$$

The state of the system is entirely specified by the $N \times D$ phase space coordinates $\widehat{\mathbf{q}}_{t}^{N} \equiv$ $\widehat{\mathbf{q}}_{t}^{(1)}, \ldots, \widehat{\mathbf{q}}_{t}^{(N)}$. In the following, it will be useful to note that the one-body term can be written as

$$
\sum_{i=1}^{N} \nabla_{i} \phi_{t \widehat{\mathbf{q}}_{t}^{(i)}}=\sum_{i=1}^{N} \nabla_{i}\left(\sum_{j=1}^{N} \phi_{t \widehat{\mathbf{q}}_{t}^{(j)}}\right) \equiv\left(\sum_{i=1}^{N} \nabla_{i}\right) \phi_{t \widehat{\mathbf{q}}_{t}^{N}},
$$

where the last equivalence defines the total external potential, $\phi_{t \widehat{\mathbf{q}}_{t}^{N}}$. The probability to find the system in a given state, $\mathbf{r}^{N}$, at time $t$ is

$$
\Psi_{t \mathbf{r}^{N}}=\left\langle\delta\left(\mathbf{r}^{(1)}-\widehat{\mathbf{q}}_{t}^{(1)}\right) \ldots \delta\left(\mathbf{r}^{(N)}-\widehat{\mathbf{q}}_{t}^{(N)}\right)\right\rangle
$$

where the brackets indicate an average over the noise, $\widehat{\boldsymbol{\xi}}_{t}^{(i)}$, and the distribution of initial conditions. That $\Psi_{t \mathbf{r}^{N}}$ is the distribution function is evident since the expectation value with respect to the noise of any function $f_{\widehat{\mathbf{q}}_{t}^{N}}$ of the stochastic variables $\widehat{\mathbf{q}}_{t}^{N}$ can be calculated as

$$
\left\langle f_{\widehat{\mathbf{q}}_{t}^{N}}\right\rangle=\int f_{\mathbf{r}^{N}} \Psi_{t_{\mathbf{r}}^{N}} d \mathbf{r}^{N}
$$

and as usual[17], the Brownian dynamics implies that the distribution satisfies the FokkerPlanck equation

$$
\frac{\partial}{\partial t} \Psi_{t \mathbf{r}^{N}}=\frac{1}{\gamma} \sum_{i=1}^{N} \nabla_{i} \cdot\left(\nabla_{i}\left(U_{\mathbf{r}^{N}}+\phi_{t \mathbf{r}^{N}}\right)+k_{B} T \nabla_{i}\right) \Psi_{t \mathbf{r}^{N}}
$$


Clearly, the distribution at time $t$ is completely determined by the interaction potential $U$, the external field $\phi_{t}$ and an initial condition that will be denoted $\Psi_{t_{0} \mathbf{r}}$. As is to be expected, the Boltzmann form $\Psi_{t^{N}} \sim \exp \left(-\beta\left(U_{\mathbf{r}^{N}}+\phi_{\mathbf{r}^{N}}\right)\right)$ is a stationary solution if the field is stationary.

In the following, square brackets will be used to indicate functional dependence so that to be precise and to indicate the full dependence of the distribution on the interaction potential, the initial condition and the external field one should write $\Psi_{t_{\mathbf{r}}}\left[U, \phi_{t} ; \Psi_{0}\right]$. Note that the spatial arguments are not indicated in the square brackets because, in principle, to know $\Psi_{t \mathbf{r}^{N}}$ at positions $\mathbf{r}^{N}$ requires knowing the various functional inputs (e.g. $U, \phi_{t}$ and $\Psi_{0}$ ) at

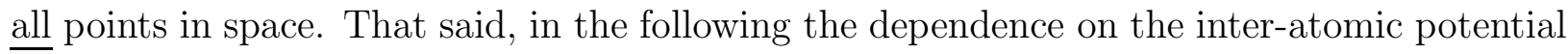
$U$ will not be explicitly indicated since it is ubiquitous, never changes and plays no role in the discussion below. We note also that SB include non-conservative forces in their analysis: although the same could be done here, we have chosen to omit them for the sake of clarity as they do not change any of the arguments to follow and only serve to complicate the expressions. We note for later purposes that, in this notation, SB write the Fokker-Planck equation in the form

$$
\frac{\partial}{\partial t} \Psi_{t \mathbf{r}^{N}}=-\frac{1}{\gamma} \sum_{i=1}^{N} \nabla_{i} \cdot\left(\mathbf{v}_{\mathbf{r}^{N}}^{(i)}\left[\phi_{t}, \Psi_{t}\right] \Psi_{t \mathbf{r}^{N}}\right)
$$

with the "velocities" $\mathbf{v}_{\mathbf{r}^{N}}^{(i)}$ and related "forces" $\mathbf{F}_{\mathbf{r}^{N}}^{\mathrm{tot}(i)}$ defined as

$$
\gamma \mathbf{v}_{\mathbf{r}^{N}}^{(i)}[\phi, \Psi]=\mathbf{F}_{\mathbf{r}^{N}}^{\mathrm{tot}(i)}[\phi, \Psi]=-\nabla_{i} U_{\mathbf{r}^{N}}-\nabla_{i} \phi_{\mathbf{r}^{N}}-k_{B} T \nabla_{i} \ln \Psi_{\mathbf{r}^{N}}
$$

The local number density (the probability to find a particle in a given infinitesimal volume) is evaluated as

$$
\rho_{\mathbf{r}}[\Psi]=\sum_{i=1}^{N} \int \delta\left(\mathbf{r}-\mathbf{r}^{(i)}\right) \Psi_{\mathbf{r}^{N}} d \mathbf{r}^{N}
$$

and, when the distribution - and so the density - is time-dependent, the number current can be defined via the continuity equation,

$$
\frac{\partial}{\partial t} \rho_{\mathbf{r}}\left[\Psi_{t}\right]=-\nabla \cdot \mathbf{J}_{\mathbf{r}}\left[\phi_{t}, \Psi_{t}\right]
$$

which, together with the Fokker-Planck equation, gives the explicit expression

$$
\mathbf{J}_{\mathbf{r}}[\phi, \Psi]=\sum_{i=1}^{N} \int \delta\left(\mathbf{r}-\mathbf{r}^{(i)}\right) \mathbf{v}_{\mathbf{r}^{N}}^{(i)}[\phi, \Psi] \Psi_{\mathbf{r}^{N}} d \mathbf{r}^{N}
$$


An important distinction that is highlighted by this notation is that between intrinsic time dependence and inherited time dependence. Inherited time dependence refers to that arising simply because a functional depends on another, time-dependent functional. For example, defining the trivial functional $I_{\mathbf{r}}[\phi]=\phi_{\mathbf{r}}$, its evaluation with a time-dependent input, e.g. $I_{\mathbf{r}}\left[\phi_{t}\right]$, has an inherited time-dependence coming solely from its dependence on the time-dependent field. Here, the functional $I$ only changes in time because its argument changes in time and otherwise, the time argument is a passive label. An example here is the current $\mathbf{J}_{\mathbf{r}}[\Psi, \phi]$ for which the time dependence is inherited from its arguments and which has no other source of time-dependence. On the other hand, the distribution $\Psi_{t^{N}}\left[\phi ; \Psi_{0}\right]$ has an intrinsic time dependence since it is the solution to the Fokker-Planck so that even if the external field is constant in time, $\Psi$ will still change in time if the initial condition is not the equilibrium distribution. On the other hand, when the external field does change with time, the distribution $\Psi_{t}$ depends on the values of the external field $\phi_{\tau \mathbf{r}}$ at all times $\tau<t$ and for this reason, one writes $\Psi_{t \mathbf{r}^{N}}\left[\phi ; \Psi_{0}\right]$ (with $\phi$ rather than $\phi_{t}$ ) because the functional acts on both the spatial coordinate and the time.

This leads to a final caveat which is important throughout the analysis in SB and below: namely, the role of causality. The Fokker-Planck equation can formally be solved as

$$
\Psi_{t \mathbf{r}^{N}}\left[\phi ; \Psi_{0}\right]=\Psi_{t_{0} \mathbf{r}^{N}}-\frac{1}{\gamma} \int_{t_{0}}^{t}\left\{\sum_{i=1}^{N} \nabla_{i} \cdot\left(\mathbf{v}_{\mathbf{r}^{N}}^{(i)}\left[\phi_{t^{\prime}}, \Psi_{t^{\prime}}\right] \Psi_{t^{\prime} \mathbf{r}^{N}}\right)\right\} d t^{\prime}
$$

where $\Psi_{t_{0} \mathbf{r}^{N}}$ represents an initial value that has to be specified. Notice that in the argument of $\Psi$ on the left hand side, we write $\phi$ rather than $\phi_{t}$ because the right hand side depends on the external field at all times prior to $t$. SB specify that the integral on the right will be understood to exclude the end-point at time $t$, so that $t_{0} \leq t^{\prime}<t$ which can be interpreted as saying that the value of the field at time $t$ is fully determined by its values at previous times corresponding, physically, to the usual understanding of causality. When it is important below to note this dependence on a field at prior times, but not on the present time, we will write, e.g., $\Psi_{t \mathbf{r}^{N}}\left[\phi_{<t} ; \Psi_{0}\right]$. 


\section{POWER FUNCTIONAL THEORY}

\section{A. Variational formulation}

The analysis of SB begins with a a quantity modeled on the Rayleigh dissipation function evaluated at a fixed time, $t$,

$$
\widehat{R}_{\mathbf{r}^{N}}\left[\widetilde{\mathbf{v}}^{N} ; \phi_{t}, \dot{\phi}_{t}, \Psi_{t}\right]=\sum_{i}\left(\frac{\gamma}{2} \widetilde{\mathbf{v}}_{\mathbf{r}^{N}}^{(i)}-\mathbf{F}_{\mathbf{r}^{N}}^{\mathrm{tot}(i)}\left[\phi_{t}, \Psi_{t}\right]\right) \cdot \widetilde{\mathbf{v}}_{\mathbf{r}^{N}}^{(i)}+\dot{\phi}_{t \mathbf{r}^{N}}
$$

where $\widetilde{\mathbf{v}}_{\mathbf{r}^{N}}^{(i)}$ is a collection of $N$ test-fields (i.e. variational fields) each a function of the $N$-positions $\mathbf{r}^{N}$. This is used to define the functional

$$
R\left[\widetilde{\mathbf{v}}^{N} ; \phi_{t}, \dot{\phi}_{t}, \Psi_{t}\right]=\int \Psi_{t \mathbf{r}^{N}} \widehat{R}_{\mathbf{r}^{N}}\left[\widetilde{\mathbf{v}}^{N} ; \phi_{t}, \dot{\phi}_{t}, \Psi_{t}\right] d \mathbf{r}^{N}
$$

which has the obvious property that its absolute, or global, minimum with respect to the test fields $\widetilde{\mathbf{v}}^{N}$ occurs at

$$
\gamma \widetilde{\mathbf{v}}_{\mathbf{r}^{N}}^{(i) \text { global-min }}\left[\phi_{t}, \Psi_{t}\right]=\mathbf{F}_{\mathbf{r}^{N}}^{\mathrm{tot}(i)}\left[\phi_{t}, \Psi_{t}\right]=-\nabla_{i} U_{\mathbf{r}^{N}}-\nabla_{i} \phi_{t \mathbf{r}^{N}}-k_{B} T \nabla_{i} \ln \Psi_{t \mathbf{r}}
$$

corresponding to the "physical" fields at this fixed time $t$ that occur in the Fokker-Planck equation as written in Eq.(17). SB then express the variational fields in terms of a new quantity, a variational distribution $\widetilde{\Psi}_{\mathbf{r}^{N}}$ via the definition

$$
\gamma \widetilde{\mathbf{v}}_{\mathbf{r}^{N}}^{(i)} \rightarrow \gamma \widetilde{\mathbf{v}}_{\mathbf{r}^{N}}^{(i)}[\phi, \widetilde{\Psi}]=\mathbf{F}_{\mathbf{r}^{N}}^{\mathrm{tot}(i)}[\phi, \widetilde{\Psi}]
$$

in terms of which the functional $R$ becomes (see Appendix A)

$R\left[\widetilde{\Psi} ; \phi_{t}, \dot{\phi}_{t}, \Psi_{t}\right]=\frac{\left(k_{B} T\right)^{2}}{2 \gamma} \int \sum_{i}\left(\nabla_{i} \ln \frac{\widetilde{\Psi}_{\mathbf{r}^{N}}}{\Psi_{t \mathbf{r}^{N}}}\right)^{2} \Psi_{t \mathbf{r}^{N}} d \mathbf{r}^{N}+\frac{1}{2} \frac{\partial}{\partial t} \Lambda\left[\phi_{t}, \Psi_{t}\right]+\frac{1}{2} \int \dot{\phi}_{t \mathbf{r}^{N}} \Psi_{t \mathbf{r}^{N}} d \mathbf{r}^{N}$

where the dot-notation indicates a time-derivative and with

$$
\Lambda[\phi, \Psi]=\int\left(k_{B} T \Psi_{\mathbf{r}^{N}} \ln \Psi_{\mathbf{r}^{N}}+\left(U_{\mathbf{r}^{N}}+\phi_{\mathbf{r}^{N}}\right) \Psi_{\mathbf{r}^{N}}\right) d \mathbf{r}^{N}
$$

Minimizing the generating function $R$ with respect to $\widetilde{\Psi}$ clearly gives $\widetilde{\Psi}^{\text {global-min }}=\Psi_{t}$, the physical distribution. As observed by SB, the quantity $\Lambda$ plays a central role in cDFT and is the same functional used by Mermin[18] to establish its fundamental theorems. Here, it plays no role in the minimization procedure and only serves to establish the value of 
generating function at its minimum. Note that at this point one has not gained much yet: the variational velocity field in Eq.(14) and the variational distribution in Eq.(17) have been introduced as extra fields, and the functional still depends on the physical but unknown distribution $\Psi_{t \mathbf{r}^{N}}$.

\section{B. Power functional}

Next, SB break up the minimization of $R$ with respect to $\widetilde{\Psi}$ into two steps as

$$
\min _{\widetilde{\Psi}} R\left[\widetilde{\Psi} ; \phi_{t}, \dot{\phi}_{t}, \Psi_{t}\right]=\min _{\bar{\rho}, \overline{\mathbf{J}}}\left\{\min _{\widetilde{\Psi} \rightarrow \bar{\rho}, \overline{\mathbf{J}}} R\left[\widetilde{\Psi} ; \phi_{t}, \dot{\phi}_{t}, \Psi_{t}\right]\right\},
$$

where the expression in curly brackets means that $R$ is first minimized with respect to the subset of possible fields $\widetilde{\Psi}$ that satisfy $\rho_{\mathbf{r}}[\widetilde{\Psi}]=\bar{\rho}_{\mathbf{r}}$ and $\mathbf{J}_{\mathbf{r}}\left[\phi_{t}, \widetilde{\Psi}\right]=\overline{\mathbf{J}}_{\mathbf{r}}$ for any given fields $\bar{\rho}_{\mathbf{r}}$ and $\overline{\mathbf{J}}_{\mathbf{r}}$. The first (inner) minimization defines a new functional

$$
\mathcal{R}\left[\bar{\rho}, \overline{\mathbf{J}} ; \phi_{t}, \dot{\phi}_{t}, \Psi_{t}\right]=\min _{\widetilde{\Psi} \rightarrow \bar{\rho}, \overline{\mathbf{J}}} R\left[\widetilde{\Psi} ; \phi_{t}, \dot{\phi}_{t}, \Psi_{t}\right]
$$

and also defines one or more fields giving the minimum, $\widetilde{\Psi}^{\min }\left[\bar{\rho}, \overline{\mathbf{J}} ; \phi_{t}, \dot{\phi}_{t}, \Psi_{t}\right]$, so that

$$
\mathcal{R}\left[\bar{\rho}, \overline{\mathbf{J}} ; \phi_{t}, \dot{\phi}_{t}, \Psi_{t}\right]=R\left[\widetilde{\Psi}^{\min }\left[\bar{\rho}, \overline{\mathbf{J}} ; \phi_{t}, \dot{\phi}_{t}, \Psi_{t}\right] ; \phi_{t}, \dot{\phi}_{t}, \Psi_{t}\right]
$$

In terms of the variational problem, it is important that $\bar{\rho}_{\mathbf{r}}$ and $\overline{\mathbf{J}}_{\mathbf{r}}$ are arbitrary fields and that even though the current corresponding to $\widetilde{\Psi}, \mathbf{J}_{\mathbf{r}}\left[\phi_{t}, \widetilde{\Psi}\right]$, carries an inherited time dependence via the external potential, this is irrelevant to the choice of $\overline{\mathbf{J}}_{\mathbf{r}}$. Instead, the time-dependence of the current manifests itself in that the resulting functional $\mathcal{R}$ carries two dependencies on $\phi_{t}$ : the explicit dependence that comes via $\Lambda$ and, now, an implicit dependence that comes from minimizing under the constraint $\mathbf{J}_{\mathbf{r}}\left[\phi_{t}, \widetilde{\Psi}\right]=\overline{\mathbf{J}}_{\mathbf{r}}$. For example, a toy model for $\mathcal{R}$, one that is perfectly consistent and illustrates this difference, is

$\mathcal{R}\left[\bar{\rho}, \overline{\mathbf{J}} ; \phi_{t}, \dot{\phi}_{t}, \Psi_{t}\right] \stackrel{?}{=} \int\left\{\left(\bar{\rho}_{\mathbf{r}}-\rho_{\mathbf{r}}\left[\Psi_{t}\right]\right)^{2}+\left(\overline{\mathbf{J}}_{\mathbf{r}}-\mathbf{J}_{\mathbf{r}}\left[\phi_{t}, \Psi_{t}\right]\right)^{2}\right\} d \mathbf{r}+\frac{1}{2} \frac{\partial}{\partial t} \Lambda\left[\phi_{t}, \Psi_{t}\right]+\frac{1}{2} \int \dot{\phi}_{t_{\mathbf{r}} N} \Psi_{t \mathbf{r}^{N}} d \mathbf{r}^{N}$

showing the explicit field dependence in $\Lambda$ as well as an implicit field dependence in $\mathbf{J}_{\mathbf{r}}$. This toy-model minimizes (in the second step) to give the correct, physical results $\bar{\rho}_{\mathbf{r}}=\rho_{\mathbf{r}}\left[\Psi_{t}\right]$ and $\overline{\mathbf{J}}_{\mathbf{r}}=\mathbf{J}_{\mathbf{r}}\left[\phi_{t}, \Psi_{t}\right]$ and this in turn (presumably) forces $\widetilde{\Psi}^{\text {global-min }}\left[\rho\left[\Psi_{t}\right], \mathbf{J}_{\mathbf{r}}\left[\phi_{t}, \Psi_{t}\right] ; \Psi_{t}, \phi_{t}, \dot{\phi}_{t}\right]=$ 
$\Psi_{t}\left[\phi_{t}, \dot{\phi}_{t}\right]$. This happens with no additional information as is the expected result of the two-step - or "constrained search" - minimization procedure since this must ultimately lead to the same result as the one-step minimization, namely the exact solution $\widetilde{\Psi}^{\text {global-min }}=\Psi_{t}$.

Following SB, the second step in the definition of the power functional is the introduction of the quantity

$$
\begin{aligned}
\bar{\Psi}_{t \mathbf{r}^{N}}[\bar{\rho}, \overline{\mathbf{J}} ; \phi, \Psi] & \equiv \Psi_{t_{0} \mathbf{r}^{N}}-\int_{t_{0}}^{t}\left\{\sum_{i=1}^{N} \nabla_{i} \cdot\left(\widetilde{\mathbf{v}}_{\mathbf{r}^{N}}^{(i) \min }\left[\phi_{t^{\prime}}\right] \widetilde{\Psi}_{t^{\prime} \mathbf{r}^{N}}^{\min }\left[\bar{\rho}_{t^{\prime}}, \overline{\mathbf{J}}_{t^{\prime}} ; \phi_{t^{\prime}}, \dot{\phi}_{t^{\prime}}, \Psi_{t^{\prime}}\right]\right)\right\} d t^{\prime}(23) \\
& =\Psi_{t_{0} \mathbf{r}^{N}-} \\
& \int_{t_{0}}^{t}\left\{\sum_{i=1}^{N} \nabla_{i} \cdot\left(\mathbf{v}_{\mathbf{r}^{N}}^{(i)}\left[\widetilde{\Psi}^{\min }\left[\bar{\rho}_{t^{\prime}}, \overline{\mathbf{J}}_{t^{\prime}} ; \phi_{t^{\prime}}, \dot{\phi}_{t^{\prime}}, \Psi_{t^{\prime}}\right], \phi_{t^{\prime}}\right] \widetilde{\Psi}_{t^{\prime} \mathbf{r}^{N}}^{\min }\left[\bar{\rho}, \overline{\mathbf{J}} ; \phi_{t^{\prime}}, \dot{\phi}_{t^{\prime}}, \Psi_{t^{\prime}}\right]\right)\right\} d t^{\prime}
\end{aligned}
$$

which is a many-particle distribution fulfilling the continuity equation if the velocities of the particles follow from the minimal trial distribution. The first line is written as in SB, Eq.(18), whereas the second is in our extended notation, showing that the minimum for the velocity fields $\widetilde{\mathbf{v}}_{\mathbf{r}^{N}}^{(i) \min }$ is determined by $\widetilde{\Psi}_{t^{\prime} \mathbf{r}^{N}}^{\min }$ through Eq. (16). Notice first that a timedependence has now been assigned to the constraints $\bar{\rho}_{t^{\prime}}, \overline{\mathbf{J}}_{t^{\prime}}$. This means that they are being specified not at a single moment but over some range of times $t_{0} \leq t \leq T$. Second, and more problematic, notice that because $\widetilde{\Psi}_{t^{\prime}}^{\min }$ is a result of Eq.(20), it has a dependence on the exact distribution $\Psi_{t^{\prime}}$ evaluated at the same time $t^{\prime}$ and so $\bar{\Psi}_{t \mathbf{r}^{N}}$ depends on the exact distribution at all earlier times. While this appears to be the implication of equations (SB11), (SB14) and (SB18) it is at odds with subsequent developments in SB since the point of this step is to eventually eliminate the exact distribution from this problem. What seems to eventually be adopted is the elimination of the exact solution $\Psi_{t}$ from the functional through replacing $\Psi_{t^{\prime}}$ by $\bar{\Psi}_{t^{\prime}}$, giving the final form of the power functional,

$$
\begin{aligned}
\overline{\mathcal{R}}\left[\bar{\rho}_{t}, \overline{\mathbf{J}}_{t} ; \phi_{t}, \dot{\phi}_{t}, \bar{\Psi}_{t}\right] & =\min _{\widetilde{\Psi} \rightarrow \bar{\rho}_{t}, \overline{\mathbf{J}}_{t}} R\left[\widetilde{\Psi} ; \phi_{t}, \dot{\phi}_{t}, \bar{\Psi}_{t}\right] \Rightarrow \widetilde{\Psi}^{\min }\left[\bar{\rho}_{t}, \overline{\mathbf{J}}_{t} ; \phi_{t}, \dot{\phi}_{t}, \bar{\Psi}_{t}\right] \\
\bar{\Psi}_{t^{N}}[\bar{\rho}, \overline{\mathbf{J}} ; \phi, \bar{\Psi}] & =\Psi_{t_{0} \mathbf{r}^{N}-} \\
& \int_{t_{0}}^{t}\left\{\sum_{i=1}^{N} \nabla_{i} \cdot\left(\mathbf{v}_{\mathbf{r}^{N}}^{(i)}\left[\widetilde{\Psi}^{\min }\left[\bar{\rho}_{t^{\prime}}, \overline{\mathbf{J}}_{t^{\prime}} ; \phi_{t^{\prime}}, \dot{\phi}_{t^{\prime}}, \bar{\Psi}_{t^{\prime}}\right], \phi_{t^{\prime}}\right] \widetilde{\Psi}_{t^{\prime} \mathbf{r}^{N}}^{\min }\left[\bar{\rho}, \overline{\mathbf{J}} ; \phi_{t^{\prime}}, \dot{\phi}_{t^{\prime}}, \bar{\Psi}_{t^{\prime}}\right]\right)\right\} d t^{\prime}
\end{aligned}
$$

The second line presents no difficulties in that evaluation of $\bar{\Psi}_{t}$ requires $\widetilde{\Psi}^{\text {min }}$ at times $t^{\prime}<t$ and this in turn only requires $\bar{\Psi}_{t^{\prime}}$ at earlier times. At this point, one can simplify the notation since everything at time $t$ is now defined in terms of $\bar{\rho}, \overline{\mathbf{J}}$ at all earlier times, so we 
will write a little more compactly

$$
\begin{aligned}
\overline{\mathcal{R}}_{t}\left[\bar{\rho}, \overline{\mathbf{J}} ; \phi_{t}, \dot{\phi}_{t}\right] & =\min _{\widetilde{\Psi} \rightarrow \bar{\rho}_{t}, \overline{\mathbf{J}}_{t}} R\left[\widetilde{\Psi} ; \phi_{t}, \dot{\phi}_{t}, \bar{\Psi}_{t}\right] \Rightarrow \widetilde{\Psi}_{t}^{\min }\left[\bar{\rho}, \overline{\mathbf{J}} ; \phi_{t}, \dot{\phi}_{t}\right] \\
\bar{\Psi}_{t \mathbf{r}^{N}}[\bar{\rho}, \overline{\mathbf{J}} ; \phi] & =\Psi_{t_{0} \mathbf{r}^{N}}-\int_{t_{0}}^{t}\left\{\sum_{i=1}^{N} \nabla_{i} \cdot\left(\mathbf{v}_{\mathbf{r}^{N}}^{(i)}\left[\widetilde{\Psi}_{t^{\prime}}^{\min }\left[\bar{\rho}, \overline{\mathbf{J}} ; \phi_{t^{\prime}}, \dot{\phi}_{t^{\prime}}\right], \phi_{t^{\prime}}\right] \widetilde{\Psi}_{t^{\prime} \mathbf{r}^{N}}^{\min }\left[\bar{\rho}, \overline{\mathbf{J}} ; \phi_{t^{\prime}}, \dot{\phi}_{t^{\prime}}\right]\right)\right\} d t^{\prime}
\end{aligned}
$$

The density implied by this $\bar{\Psi}$ is now easily evaluated from the last of these with the result

$$
\rho_{\mathbf{r}}\left[\bar{\Psi}_{t}\right]=\rho_{\mathbf{r}}\left[\Psi_{t_{0}}\right]-\int_{t_{0}}^{t} \nabla \cdot \overline{\mathbf{J}}_{t^{\prime} \mathbf{r}} d t^{\prime}
$$

which follows because, by definition, $\widetilde{\Psi}_{t^{\prime}}^{\min }[\bar{\rho}, \overline{\mathbf{J}}]$ is precisely the distribution that implies the current $\overline{\mathbf{J}}_{t^{\prime}}$ while minimizing the generating function. So, the implied density $\rho_{\mathbf{r}}\left[\bar{\Psi}_{t}\right]$ satisfies a continuity equation with respect to the current $\overline{\mathbf{J}}_{t \mathbf{r}}$. One might have expected that $\rho_{\mathbf{r}}\left[\bar{\Psi}_{t}\right]$ should simply be $\bar{\rho}_{t \mathbf{r}}$ but this is clearly not the case since, at this point, the temporal evolution of the fields $\bar{\rho}_{t \mathbf{r}}$ and $\overline{\mathbf{J}}_{t \mathbf{r}}$ are completely independent. Indeed, one also sees that in general $\mathbf{J}_{\mathbf{r}}\left[\bar{\Psi}_{t} ; \phi_{t}\right] \neq \overline{\mathbf{J}}_{t \mathbf{r}}$, i.e., the one-particle current from the distribution $\bar{\Psi}$ is not the constraint current. This turns out to be important.

\section{Problems interpreting the power functional}

In $\mathrm{SB}$, one of the main results is that the power functional has a Legendre structure with, e.g., the density $\bar{\rho}_{t}$ and $\phi_{t}$ playing the role of conjugate variables thus mirroring the relation between the density and the external field in equilibrium DFT. In order to verify this, we now turn to an examination of the power functional with the aim of making the dependencies on the field at time $t$ explicit. Starting from the SB functional (Eq. (14)), performing the first minimization with the replacement $\Psi_{t \mathbf{r}^{N}} \rightarrow \bar{\Psi}_{t \mathbf{r}^{N}}$ (as described) and following the steps detailed in Appendix $\mathrm{A}$ gives

$$
\begin{aligned}
\overline{\mathcal{R}}_{t}\left[\bar{\rho}, \overline{\mathbf{J}} ; \phi_{t}, \dot{\phi}_{t}\right] & =\frac{\left(k_{B} T\right)^{2} \gamma}{2} \int \bar{\Psi}_{t \mathbf{r}^{N}}[\bar{\rho}, \overline{\mathbf{J}} ; \phi]\left(\sum_{i}\left(\nabla_{i} \ln \frac{\bar{\Psi}_{t \mathbf{r}^{N}}[\bar{\rho}, \overline{\mathbf{J}} ; \phi]}{\widetilde{\Psi}_{t \mathbf{r}^{N}}^{\min }\left[\bar{\rho}, \overline{\mathbf{J}} ; \phi_{t}, \dot{\phi} t_{t}\right]}\right)^{2}\right) d \mathbf{r}^{N} \\
& -\frac{1}{2 \gamma} \int \bar{\Psi}_{t^{N}}[\bar{\rho}, \overline{\mathbf{J}} ; \phi] \sum_{i}\left(\mathbf{F}_{\mathbf{r}^{N}}^{\mathrm{tot}(i)}\left[0, \bar{\Psi}_{t}\right]\right)^{2} d \mathbf{r}^{N} \\
& -\frac{1}{2 \gamma} \int \rho_{\mathbf{r}}\left[\bar{\Psi}_{t}\right]\left(\nabla \phi_{t \mathbf{r}}\right)^{2} d \mathbf{r}+\int \mathbf{J}_{\mathbf{r}}\left[0, \bar{\Psi}_{t}\right] \cdot\left(\nabla \phi_{\mathbf{r}}\right) d \mathbf{r}+\int \dot{\phi}_{t \mathbf{r}} \rho_{\mathbf{r}}\left[\bar{\Psi}_{t}\right] d \mathbf{r} .
\end{aligned}
$$


Here, all explicit dependence on the external potential at time $t$ has been exposed. SB argue that this can be written as (see SB Eq.(25))

$$
\overline{\mathcal{R}}_{t}^{(S B)}[\bar{\rho}, \overline{\mathbf{J}} ; \phi, \dot{\phi}]=W_{t}^{(S B)}[\bar{\rho}, \overline{\mathbf{J}} ; \phi, \dot{\phi}]-\int \overline{\mathbf{J}}_{\mathbf{r}} \cdot\left(\nabla \phi_{t \mathbf{r}}\right) d \mathbf{r}+\int \dot{\phi}_{t \mathbf{r}} \bar{\rho}_{t \mathbf{r}} d \mathbf{r}
$$

where $W_{t}^{(S B)}$ does not depend on the external field at time $t$ but only at earlier times. This form is crucial later on in SB for identifying a truly intrinsic functional of the dissipated power, similar to the intrinsic free energy functional in equilibrium DFT. Our form (27) reveals three difficulties with that interpretation. The first is that in Eq. (27) one cannot guarantee that $\widetilde{\Psi}_{t_{\mathbf{r}}}^{\min }$ does not depend on the external field at time $t$. Indeed, the global minimum of $R$ certainly does (see Eq. (15)) so it is entirely possible that the constrained minimum $\widetilde{\Psi}_{t \mathbf{r}^{N}}^{\min }$ does as well. The second is that one sees in Eq. (27) an explicit, quadratic dependence on the field at time $t$, which is ignored in $\mathrm{SB}$. This seems to be because SB switch between the representation of the variational field as $\widetilde{\Psi}$ and the original formulation in terms of $\widetilde{\mathbf{v}}^{N}$ and in terms of the latter there is no quadratic term. However, formulating everything in terms of the velocities is not possible at this point as the definition of $\bar{\Psi}_{t \mathbf{r}^{N}}$, see Eqs. (24) and (25), involves both $\mathbf{v}_{\mathbf{r}^{N}}^{(i)}\left[\widetilde{\Psi}_{t^{\prime}}^{\min } \ldots\right]$, which could indeed be replaced by $\widetilde{\mathbf{v}}_{\mathbf{r}^{N}}^{(i) \min }$ but also explicitly $\widetilde{\Psi}_{t^{\prime}}^{\min }$, which cannot. The third problem is that the term involving the gradient of the field in the last line of Eq.(27) is written in terms of $\mathbf{J}_{\mathbf{r}}\left[0, \bar{\Psi}_{t}\right]$ and $\rho_{\mathbf{r}}\left[\bar{\Psi}_{t}\right]$ rather than $\mathbf{J}_{\mathbf{r}}\left[\widetilde{\Psi}_{t}^{\min } ; \phi_{t}\right]=\overline{\mathbf{J}}_{t \mathbf{r}}$ and $\rho_{\mathbf{r}}\left[\widetilde{\Psi}_{t}^{\min }\right]=\bar{\rho}_{t \mathbf{r}}$, as tacitly assumed in SB. This means, e.g., that SB Eq.(26),

$$
\frac{\delta}{\delta \dot{\phi}_{t \mathbf{r}}} \overline{\mathcal{R}}_{t}^{(S B)}[\bar{\rho}, \overline{\mathbf{J}} ; \phi, \dot{\phi}]=\bar{\rho}_{t \mathbf{r}}
$$

in which $\overline{\mathcal{R}}_{t}^{(S B)}$ is meant to act as a generator for the density does not hold here. Finally, we observe that several of these problems can be traced to the fact that $\widetilde{\Psi}_{t}^{\text {min }}$ and $\bar{\Psi}_{t}$ are not the same quantities - a fact that we will exploit below to try to repair these problems.

\section{Using the power functional}

Before exploring modifications of PFT, it is worthwhile to recall the final purpose. SB eventually introduce an ansatz for the power functional which is (SB Eq.(30))

$$
\overline{\mathcal{R}}_{t}^{(S B)}[\bar{\rho}, \overline{\mathbf{J}} ; \phi, \dot{\phi}]=P_{t}[\bar{\rho}, \overline{\mathbf{J}}]+\int \overline{\mathbf{J}}_{t \mathbf{r}} \cdot \nabla \frac{\delta F\left[\bar{\rho}_{t}\right]}{\delta \bar{\rho}_{t \mathbf{r}}} d \mathbf{r}-\int \overline{\mathbf{J}}_{t \mathbf{r}} \cdot\left(\nabla \phi_{t \mathbf{r}}\right) d \mathbf{r}+\int \dot{\phi}_{t \mathbf{r}} \bar{\rho}_{t \mathbf{r}} d \mathbf{r} .
$$


where $P_{t}[\bar{\rho}, \overline{\mathbf{J}}]=W_{t}^{(S B)}[\bar{\rho}, \overline{\mathbf{J}}]-\int \overline{\mathbf{J}}_{t \mathbf{r}} \cdot \nabla \frac{\delta F\left[\bar{\rho}_{t}\right]}{\delta \bar{\rho}_{t \mathbf{r}}} d \mathbf{r}$ is the intrinsic functional of dissipated power. This is then used to complete the minimization procedure defined in Eq.(19) giving the equations

$$
\begin{aligned}
& \frac{\delta}{\delta \overline{\mathbf{J}}_{t \mathbf{r}}} \overline{\mathcal{R}}_{t}^{(S B)}[\bar{\rho}, \overline{\mathbf{J}} ; \phi, \dot{\phi}]=0 \\
& \frac{\delta}{\delta \bar{\rho}_{t \mathbf{r}}} \overline{\mathcal{R}}_{t}^{(S B)}[\bar{\rho}, \overline{\mathbf{J}} ; \phi, \dot{\phi}]=0
\end{aligned}
$$

however such an interpretation is untenable as it leads to unphysical results (see Appendix B). In fact, SB say at this point that a Lagrange multiplier should be introduced prior to minimization so as to enforce the continuity equation relating the density and current. This statement is problematic for three reasons. First, as illustrated above when discussing the toy model, Eq.(22), the continuity equation is already implicit in the formalism since it reproduces the exact distribution from which the continuity equation for the density is automatically valid. Second, this ad-hoc modification would not be the minimization that was defined in Eq.(19) and that has been used throughout the analysis. Third, if $\bar{\rho}_{\text {tr }}$ and $\overline{\mathbf{J}}_{t \mathbf{r}}$ are related by the continuity equation then they could never have been treated as independent constraints - since the density is then fully determined by the temporal history of the current - and the original split of the minimization should have taken the form

$$
\min _{\widetilde{\Psi}} R\left[\widetilde{\Psi} ; \phi_{t}, \dot{\phi}_{t}, \Psi_{t}\right]=\min _{\overline{\mathbf{J}}}\left\{\min _{\widetilde{\Psi} \rightarrow \overline{\mathbf{J}}} R\left[\widetilde{\Psi} ; \phi_{t}, \dot{\phi}_{t}, \Psi_{t}\right]\right\},
$$

with no density constraint at all and so leaving only the variational equation with respect to the current. In such a case, with no variation with respect to the density, the relationship with classical DFT (as the equilibrium limit) becomes less clear: it is relegated to the statement - based on the ansatz above - that the current is zero, and so the density stationary in time, if the equilibrium condition

$$
\nabla \frac{\delta F\left[\bar{\rho}_{t}\right]}{\delta \bar{\rho}_{t \mathbf{r}}}=\nabla \mu=0
$$

for some constant $\mu$ holds.

\section{A VARIATION ON PFT}

The discussion above suggests that several of the problems identified can be addressed with a few modifications of the theory, in particular the use of the variational velocities 
rather than the variational distribution and imposing a constraint on the current but not on the density. Since the distribution is required in the definition of the current, some replacement for the variational distribution $\widetilde{\Psi}$ must be found. Recalling as one source of problems that $\mathbf{J}_{\mathbf{r}}\left[\bar{\Psi}_{t} ; \phi_{t}\right] \neq\left(\overline{\mathbf{J}}_{t \mathbf{r}}=\mathbf{J}_{\mathbf{r}}\left[\widetilde{\Psi}_{t}^{\text {min }} ; \phi_{t}\right]\right)$, we begin with a redefinition of $\bar{\Psi}_{t}$ as satisfying the equation

$$
\frac{\partial}{\partial t} \bar{\Psi}_{t \mathbf{r}^{N}}\left[\widetilde{\mathbf{v}}^{N} ; \phi\right]=-\sum_{i} \nabla_{i} \cdot \widetilde{\mathbf{v}}_{t \mathbf{r}^{N}}^{(i)} \bar{\Psi}_{t \mathbf{r}^{N}}\left[\widetilde{\mathbf{v}}^{N} ; \phi\right], \quad \bar{\Psi}_{t_{0} \mathbf{r}^{N}}=\Psi_{t_{0} \mathbf{r}^{N}}
$$

for any set of variational velocities $\widetilde{\mathbf{v}}_{t_{\mathbf{r}}}^{(i)}$ specified for all relevant times. This describes the time evolution of the many-body distribution due to an arbitrary velocity field, the variational field $\widetilde{\mathbf{v}}^{N}$, with initial condition $\Psi_{t_{0} \mathbf{r}^{N}}$ that is the same initial condition as for the exact distribution. This is of course equivalent to the integral form

$$
\bar{\Psi}_{t \mathbf{r}^{N}}\left[\widetilde{\mathbf{v}}^{N} ; \phi\right]=\Psi_{t_{0} \mathbf{r}^{N}}-\int_{t_{0}}^{t}\left\{\sum_{i=1}^{N} \nabla_{i} \cdot\left(\widetilde{\mathbf{v}}_{t^{\prime} \mathbf{r}^{N}}^{(i)} \bar{\Psi}_{t^{\prime} \mathbf{r}^{N}}\left[\widetilde{\mathbf{v}}^{N} ; \phi\right]\right)\right\} d t^{\prime} .
$$

We keep the original definition of $R$, Eq.(14), evaluated using $\bar{\Psi}$ in place of the exact distribution giving

$$
R\left[\widetilde{\mathbf{v}}^{N} ; \phi_{t}, \dot{\phi}_{t}, \bar{\Psi}_{t}\right]=\int_{t \mathbf{r}^{N}} \bar{\Psi}_{t \mathbf{r}^{N}} \sum_{i}\left(\frac{\gamma}{2} \widetilde{\mathbf{v}}_{\mathbf{r}^{N}}^{(i)}-\mathbf{F}_{\mathbf{r}^{N}}^{\mathrm{tot}(i)}\left[\phi_{t}, \bar{\Psi}_{t}\right]\right) \cdot \widetilde{\mathbf{v}}_{\mathbf{r}^{N}}^{(i)} d \mathbf{r}^{N}+\int \bar{\Psi}_{t \mathbf{r}^{N}} \dot{\phi}_{t \mathbf{r}^{N}} d \mathbf{r}^{N}
$$

so that minimization will now give

$$
\widetilde{\mathbf{v}}_{\mathbf{r}^{N}}^{(i) \text { global-min }}\left[\phi_{t}, \bar{\Psi}_{t}\right]=\frac{1}{\gamma} \mathbf{F}_{\mathbf{r}^{N}}^{\mathrm{tot}(i)}\left[\phi_{t}, \bar{\Psi}_{t}\right]=-\nabla_{i} U_{\mathbf{r}^{N}}-\nabla_{i} \phi_{t \mathbf{r}^{N}}-k_{B} T \nabla_{i} \ln \bar{\Psi}_{t \mathbf{r}^{N}} .
$$

When this is substituted into the evolution equation, Eq.(34), it becomes the Fokker-Planck equation and so, with the exact initial condition, one recovers the exact distribution for the system thus demonstrating that this is an exact reformulation of the problem.

The power functional is now defined using only a constraint on the current as

$$
\mathcal{R}_{t}[\overline{\mathbf{J}} ; \phi, \dot{\phi}]=\min _{\mathbf{J}\left[\widetilde{\mathbf{v}}^{N} ; \bar{\Psi}_{t}\right]=\overline{\mathbf{J}}_{t}} R\left[\widetilde{\mathbf{v}}^{N} ; \phi_{t}, \dot{\phi}_{t}, \bar{\Psi}_{t}^{\min }\right]=R\left[\widetilde{\mathbf{v}}_{t}^{N \min }[\overline{\mathbf{J}} ; \phi] ; \phi_{t}, \dot{\phi}_{t}, \bar{\Psi}_{t}^{\min }\left[\overline{\mathbf{J}}_{<t} ; \phi, \dot{\phi}\right]\right]
$$

so here, the current is evaluated from the usual definition, Eq.(11), using $\widetilde{\mathbf{v}}^{N}$ and $\bar{\Psi}_{t}$ as inputs and its value is constrained to be the specified $\overline{\mathbf{J}}_{t}$. At fixed time $t$, the minimization is only a minimization with respect to $\widetilde{\mathbf{v}}^{N}$ at time $t$ since $\bar{\Psi}_{t}^{\min }$ only depends on $\widetilde{\mathbf{v}}_{t^{\prime}}^{N m i n}$ at earlier times $t^{\prime}<t$. Also, we have written $\bar{\Psi}_{t}^{\min }\left[\overline{\mathbf{J}}_{<t}\right]$ (equivalent to $\bar{\Psi}_{t}^{\min }\left[\widetilde{\mathbf{v}}_{<t}^{N \min }\right]$ ) because $\bar{\Psi}_{t}$ depends 
on $\widetilde{\mathbf{v}}_{t^{\prime} \mathbf{r}^{N}}^{N}$ for earlier times and so we replace these with the (independently determined) $\widetilde{\mathbf{v}}_{t^{\prime} \mathbf{r}^{N}}^{N \min }[\overline{\mathbf{J}}]$ leaving only an over-all dependence on the current temporal history. Analyzing this as before, one finds the power functional

$\mathcal{R}_{t}\left[\overline{\mathbf{J}}_{t} ; \phi_{t}, \dot{\phi}_{t}\right]=\int \bar{\Psi}_{t \mathbf{r}^{N}}^{\min }\left[\overline{\mathbf{J}}_{<t} ; \phi, \dot{\phi}\right]\left(\sum_{i}\left(\frac{\gamma}{2} \widetilde{\mathbf{v}}_{t \mathbf{r}^{N}}^{(i) \min }[\overline{\mathbf{J}}, \phi, \dot{\phi}]-\mathbf{F}_{\mathbf{r}^{N}}^{\operatorname{tot}(i)}\left[0, \bar{\Psi}_{t}^{\min }\right]\right) \cdot \widetilde{\mathbf{v}}_{t \mathbf{r}^{N}}^{(i) \min }[\overline{\mathbf{J}}, \phi, \dot{\phi}]\right) d \mathbf{r}^{N}$

$$
-\int \overline{\mathbf{J}}_{t \mathbf{r}} \cdot\left(\nabla \phi_{t \mathbf{r}}\right) d \mathbf{r}+\int \dot{\phi}_{t \mathbf{r}} \rho_{\mathbf{r}}\left[\bar{\Psi}_{t}^{\min }\right] d \mathbf{r}
$$

which has the form of SB Eq. (25) consisting of the sum of a term independent of the field plus linear dependencies on the field written in terms of the current $\overline{\mathbf{J}}$ and the corresponding density. Thus, some of the structural problems discussed above have been resolved, although once again one cannot say at this point that $\widetilde{\mathbf{v}}_{t \mathbf{r}^{N}}^{(i) \min }$ is independent of the field at time $t$.

One could continue by introducing an ansatz for the power functional as in SB, but here we are able to do much more because it turns out that - unlike in SB - the power functional can be evaluated exactly. Let us return to the basic definition in Eq.(38) and note that the constrained minimization can be formulated using a Lagrange parameter (really, a vector field $\boldsymbol{\lambda}_{\mathbf{r}}$ ) by first defining the Lagrangian

$$
L\left[\widetilde{\mathbf{v}}^{N} ; \phi_{t}, \dot{\phi}_{t}, \bar{\Psi}_{t}\right]=R\left[\widetilde{\mathbf{v}}^{N} ; \phi_{t}, \dot{\phi}_{t}, \bar{\Psi}_{t}\right]-\int \boldsymbol{\lambda}_{\mathbf{r}} \cdot\left(\overline{\mathbf{J}}_{t \mathbf{r}}-\mathbf{J}_{\mathbf{r}}\left[\widetilde{\mathbf{v}}^{N} ; \phi_{t}, \bar{\Psi}_{t}\right]\right) d \mathbf{r}
$$

and then minimizing by solving

$$
\begin{aligned}
& 0=\frac{\delta}{\delta \widetilde{\mathbf{v}}_{\mathbf{r}}^{(i)}} L\left[\widetilde{\mathbf{v}}^{N} ; \phi_{t}, \dot{\phi}_{t}, \bar{\Psi}_{t}^{\min }\right] \\
& 0=\frac{\delta}{\delta \boldsymbol{\lambda}_{\mathbf{r}}} L\left[\widetilde{\mathbf{v}}^{N} ; \phi_{t}, \dot{\phi}_{t}, \bar{\Psi}_{t}^{\min }\right]
\end{aligned}
$$

which gives, since $R$ is quadratic in the velocities,

$$
\begin{aligned}
& 0=\bar{\Psi}_{t \mathbf{r}^{N}}\left[\widetilde{\mathbf{v}}_{t}^{N}, \phi\right]\left(\gamma \widetilde{\mathbf{v}}_{t \mathbf{r}^{N}}^{(i)}-\mathbf{F}_{\mathbf{r}^{N}}^{\mathrm{tot}(i)}\left[\phi_{t}, \bar{\Psi}_{t}^{\min }\right]\right)+\int\left(\frac{\delta}{\delta \widetilde{\mathbf{v}}_{\mathbf{r}}^{(i)}} \mathbf{J}_{\mathbf{r}}\left[\widetilde{\mathbf{v}}^{N} ; \phi_{t}, \bar{\Psi}_{t}^{\min }\right]\right) \cdot \boldsymbol{\lambda}_{\mathbf{r}} d \mathbf{r} \\
& 0=\overline{\mathbf{J}}_{t \mathbf{r}}-\mathbf{J}_{\mathbf{r}}\left[\widetilde{\mathbf{v}}^{N} ; \phi_{t}, \bar{\Psi}_{t}^{\min }\right]
\end{aligned}
$$

Now, it is straightforward to evaluate

$$
\int\left(\frac{\delta}{\delta \widetilde{\mathbf{v}}_{\mathbf{r}}^{(i)}} \mathbf{J}_{\mathbf{r}}\left[\widetilde{\mathbf{v}}^{N} ; \phi_{t}, \bar{\Psi}_{t}^{\min }\right]\right) \cdot \boldsymbol{\lambda}_{\mathbf{r}} d \mathbf{r}=\int \boldsymbol{\lambda}_{\mathbf{r}} \delta\left(\mathbf{r}-\mathbf{r}_{i}\right) \bar{\Psi}_{t \mathbf{r}^{N}}^{\min } d \mathbf{r}=\boldsymbol{\lambda}_{\mathbf{r}_{i}} \bar{\Psi}_{t \mathbf{r}^{N}}^{\min }
$$


so that the system of equations becomes

$$
\begin{aligned}
& 0=\bar{\Psi}_{t \mathbf{r}^{N}}^{\min }\left[\widetilde{\mathbf{v}}_{t}^{N}, \phi\right]\left(\gamma \widetilde{\mathbf{v}}_{t \mathbf{r}^{N}}^{(i)}-\mathbf{F}_{\mathbf{r}^{N}}^{\operatorname{tot}(i)}\left[\phi_{t}, \bar{\Psi}_{t}^{\min }\right]\right)+\boldsymbol{\lambda}_{\mathbf{r}_{i}} \bar{\Psi}_{t \mathbf{r}^{N}}^{\min }\left[\widetilde{v}_{t}^{N}, \phi\right] \\
& 0=\overline{\mathbf{J}}_{t \mathbf{r}}-\mathbf{J}_{\mathbf{r}}\left[\widetilde{\mathbf{v}}^{N} ; \phi_{t}, \bar{\Psi}_{t}^{\min }\right] .
\end{aligned}
$$

The first line gives

$$
\widetilde{\mathbf{v}}_{t \mathbf{r}^{N}}^{(i) \min }[\overline{\mathbf{J}}, \phi, \dot{\phi}]=\frac{1}{\gamma} \mathbf{F}_{\mathbf{r}^{N}}^{\mathrm{tot}(i)}\left[\phi_{t}, \bar{\Psi}_{t}^{\min }\right]-\frac{1}{\gamma} \boldsymbol{\lambda}_{\mathbf{r}_{i}}
$$

while the second then evaluates to

$$
\overline{\mathbf{J}}_{t \mathbf{r}}=\mathbf{J}_{\mathbf{r}}\left[\widetilde{\mathbf{v}}^{N \min } ; \phi_{t}, \bar{\Psi}_{t}^{\min }\right]=\mathbf{J}_{\mathbf{r}}\left[\phi_{t}, \bar{\Psi}_{t}^{\min }\right]-\frac{1}{\gamma} \boldsymbol{\lambda}_{\mathbf{r}} \rho_{\mathbf{r}}\left[\bar{\Psi}_{t}^{\min }\right]
$$

so that the solution to the minimization problem is

$$
\begin{gathered}
\boldsymbol{\lambda}_{\mathbf{r}}^{\min }=\gamma \frac{\mathbf{J}_{\mathbf{r}}\left[\phi_{t}, \bar{\Psi}_{t}^{\min }\right]-\overline{\mathbf{J}}_{t \mathbf{r}}}{\rho_{\mathbf{r}}\left[\bar{\Psi}_{t}^{\min }\right]} \\
\widetilde{\mathbf{v}}_{t^{N}}^{(i) \min }[\overline{\mathbf{J}}, \phi, \dot{\phi}]=\frac{1}{\gamma} \mathbf{F}_{\mathbf{r}^{N}}^{\mathrm{tot}(i)}\left[\phi_{t}, \bar{\Psi}_{t}^{\min }\right]-\frac{\mathbf{J}_{\mathbf{r}_{i}}\left[\phi_{t}, \bar{\Psi}_{t}^{\min }\right]-\overline{\mathbf{J}}_{t \mathbf{r}_{i}}}{\rho_{\mathbf{r}_{i}}\left[\bar{\Psi}_{t}^{\min }\right]} .
\end{gathered}
$$

Using this to evaluate the power functional gives

$$
\begin{aligned}
\mathcal{R}_{t}\left[\overline{\mathbf{J}}_{t} ; \phi_{t}, \dot{\phi}_{t}\right]= & -\frac{1}{2 \gamma} \int \bar{\Psi}_{t \mathbf{r}^{N}}^{\min } \sum_{i}\left(\mathbf{F}_{\mathbf{r}^{N}}^{\mathrm{tot}(i)}\left[\phi_{t}, \bar{\Psi}_{t}^{\min }\right]\right)^{2} d \mathbf{r}^{N}+\frac{\gamma}{2} \int \frac{\left(\overline{\mathbf{J}}_{t \mathbf{r}}-\mathbf{J}_{\mathbf{r}}\left[\phi_{t}, \bar{\Psi}_{t}^{\min }\right]\right)^{2}}{\rho_{\mathbf{r}}\left[\bar{\Psi}_{t}^{\min }\right]} d \mathbf{r} \\
& +\int \dot{\phi}_{t \mathbf{r}} \rho_{\mathbf{r}}\left[\bar{\Psi}_{t}^{\min }\right] d \mathbf{r} .
\end{aligned}
$$

The dependence on the external field $\phi_{t}$ appearing in $\mathbf{F}_{\mathbf{r}^{N}}^{\mathrm{tot}(i)}\left[\phi_{t}, \bar{\Psi}_{t}^{\min }\right]$ and $\mathbf{J}_{\mathbf{r}}\left[\phi_{t}, \bar{\Psi}_{t}^{\min }\right]$ can be explicitly taken out and the functional is rewritten as

$$
\begin{aligned}
\mathcal{R}_{t}\left[\overline{\mathbf{J}}_{t} ; \phi_{t}, \dot{\phi}_{t}\right]= & -\frac{1}{2 \gamma} \int \bar{\Psi}_{t \mathbf{r}^{N}}^{\min } \sum_{i}\left(\mathbf{F}_{\mathbf{r}^{N}}^{\mathrm{tot}(i)}\left[0, \bar{\Psi}_{t}^{\min }\right]\right)^{2} d \mathbf{r}^{N}+\frac{\gamma}{2} \int \frac{\left(\overline{\mathbf{J}}_{t \mathbf{r}}-\mathbf{J}_{\mathbf{r}}\left[0, \bar{\Psi}_{t}^{\min }\right]\right)^{2}}{\rho_{\mathbf{r}}\left[\bar{\Psi}_{t}^{\min }\right]} d \mathbf{r} \\
& -\int \overline{\mathbf{J}}_{t \mathbf{r}} \cdot\left(\nabla \phi_{t \mathbf{r}}\right) d \mathbf{r}+\int \dot{\phi}_{t \mathbf{r}} \rho_{\mathbf{r}}\left[\bar{\Psi}_{t}^{\mathrm{min}}\right] d \mathbf{r}
\end{aligned}
$$

thus explicitly showing the structure discussed in SB whereby the external field contributions at time $t$ appear linearly. Finally, we note that the density automatically satisfies the 
continuity equation since

$$
\begin{aligned}
\frac{\partial}{\partial t} \rho_{\mathbf{r}}\left[\bar{\Psi}_{t}^{\min }\right] & =\int \frac{\partial}{\partial t} \bar{\Psi}_{t \mathbf{r}^{N}}^{\min } \sum_{i} \delta\left(\mathbf{r}-\mathbf{r}_{i}\right) d \mathbf{r}^{N} \\
& =-\int \sum_{i} \delta\left(\mathbf{r}-\mathbf{r}_{i}\right) \nabla_{i} \cdot\left(\widetilde{\mathbf{v}}_{t \mathbf{r}^{N}}^{(i) \min } \bar{\Psi}_{t \mathbf{r}^{N}}^{\min }\right) d \mathbf{r}^{N} \\
& =-\nabla \cdot \int \sum_{i} \delta\left(\mathbf{r}-\mathbf{r}_{i}\right) \widetilde{\mathbf{v}}_{t \mathbf{r}^{N}}^{(i) \min } \bar{\Psi}_{t \mathbf{r}^{N}}^{\min } d \mathbf{r}^{N} \\
& =-\nabla \cdot \overline{\mathbf{J}}_{t \mathbf{r}}
\end{aligned}
$$

by definition of $\widetilde{\mathbf{v}}_{t \mathbf{r}^{N}}^{(i) \min }$. This shows that we can replace $\rho_{\mathbf{r}}\left[\bar{\Psi}_{t}^{\min }\right]$ by $\rho_{t \mathbf{r}}[\overline{\mathbf{J}}]$ since the current (and an initial condition) completely determines the density. We can interpret this construction as follows: $\mathcal{R}_{t}\left[\overline{\mathbf{J}}_{t} ; \phi_{t}, \dot{\phi}_{t}\right]$ is a functional for the power in a system which is described by a certain time-dependent current $\overline{\mathbf{J}}_{t}$. The associated many-body distribution $\bar{\Psi}_{t}^{\text {min }}\left[\overline{\mathbf{J}}_{<t}\right]$ is consistent with this current (i.e. they are related by the usual definition) and the corresponding local density fulfills the continuity with $\overline{\mathbf{J}}_{t}$ as the material-transporting current. Upon minimization with respect to $\overline{\mathbf{J}}_{t}$, under the usual constraint of causality, the minimal current $\overline{\mathbf{J}}_{t}^{\text {min }}$ and $\bar{\Psi}_{t}^{\text {min }}\left[\overline{\mathbf{J}}_{<t}\right]$ become the exact physical solutions corresponding to the applied field $\phi_{t}$.

\section{A. Pessimistic interpretation of these results}

Following SB, the power functional is to be minimized with respect to the current so as to get the minimizing current. Here, this is trivial and results in

$$
\overline{\mathbf{J}}_{t \mathbf{r}}^{\min }=\mathbf{J}_{\mathbf{r}}\left[\phi_{t}, \bar{\Psi}_{t}^{\min }\left[\overline{\mathbf{J}}_{<t}\right]\right]
$$

and from Eq.(47), one then has for the variational velocities

$$
\widetilde{\mathbf{v}}_{t \mathbf{r}^{N}}^{(i) \min }\left[\overline{\mathbf{J}}_{t \mathbf{r}}^{\min }, \phi, \dot{\phi}\right]=\frac{1}{\gamma} \mathbf{F}_{\mathbf{r}^{N}}^{\mathrm{tot}(i)}\left[\phi_{t}, \bar{\Psi}_{t}^{\min }\right] .
$$

As demonstrated at the start of this section (see Eq. (34)), this simply implies that $\bar{\Psi}_{t}^{\text {min }}$ satisfies the original Fokker-Planck equation and, so, is the exact distribution. One arrives at the same conclusion by recognizing that the solution to Eq. (51) for the current at time $t$ depends on the current at all earlier times, and for finding a self-consistent solution 
$\left(\overline{\mathbf{J}}_{<t}=\overline{\mathbf{J}}_{<t}^{\min }\right)$ one has to solve Eq. (51) at all earlier times successively. This amounts to nothing but the solution of the original Fokker-Planck equation.

Without the freedom to introduce an ansatz by hand, the formalism just reduces to the original exact result. There seems, therefore, to be no advantage to this development since, while one could introduce one of the usual approximations (e.g. local equilibrium) at any point, there seems no particular rationale to do so as opposed to, e.g., simply inserting such an ansatz directly into the continuity equation for the density (as is done in heuristic derivations of DDFT[12]).

\section{B. An optimistic interpretation}

A somewhat different interpretation of the formalism is possible upon consideration of the evolution equation for $\bar{\Psi}_{t}^{\text {min }}$, evaluated with the velocities constrained by the current, Eq.(47),

$$
\begin{aligned}
\frac{\partial}{\partial t} \bar{\Psi}_{t \mathbf{r}^{N}}^{\min }\left[\overline{\mathbf{J}}_{<t}, \phi\right]= & -\sum_{i} \nabla_{i} \cdot \frac{1}{\gamma} \mathbf{F}_{\mathbf{r}^{N}}^{\mathrm{tot}(i)}\left[\phi_{t}, \bar{\Psi}_{t}^{\min }\right] \bar{\Psi}_{t \mathbf{r}^{N}}^{\min }\left[\overline{\mathbf{J}}_{<t}, \phi\right] \\
& +\sum_{i} \nabla_{i} \cdot\left(\frac{\mathbf{J}_{\mathbf{r}_{i}}\left[\bar{\Psi}_{t}^{\min }, \phi_{t}\right]-\overline{\mathbf{J}}_{t \mathbf{r}_{i}}}{\rho_{t \mathbf{r}_{i}}[\overline{\mathbf{J}}]} \bar{\Psi}_{t \mathbf{r}^{N}}^{\min }\left[\overline{\mathbf{J}}_{<t}, \phi\right]\right), \bar{\Psi}_{t_{0} \mathbf{r}^{N}}^{\min }=\Psi_{t_{0} \mathbf{r}^{N}}
\end{aligned}
$$

and it is straightforward to show that the explicit contribution of the external field from $\mathbf{F}_{\mathbf{r}^{N}}^{\text {tot }}$ cancels on the right hand side leaving

$$
\begin{aligned}
\frac{\partial}{\partial t} \bar{\Psi}_{t \mathbf{r}^{N}}^{\min }\left[\overline{\mathbf{J}}_{<t}, \phi\right]= & -\sum_{i} \nabla_{i} \cdot \frac{1}{\gamma} \mathbf{F}_{\mathbf{r}^{N}}^{\mathrm{tot}(i)}\left[0, \bar{\Psi}_{t}^{\min }\right] \bar{\Psi}_{t \mathbf{r}^{N}}^{\min }\left[\overline{\mathbf{J}}_{<t}, \phi\right] \\
& +\sum_{i} \nabla_{i} \cdot\left(\frac{\mathbf{J}_{\mathbf{r}_{i}}\left[0, \bar{\Psi}_{t}^{\min }\right]-\overline{\mathbf{J}}_{t \mathbf{r}_{i}}}{\rho_{t \mathbf{r}_{i}}[\overline{\mathbf{J}}]} \bar{\Psi}_{t \mathbf{r}^{N}}^{\min }\left[\overline{\mathbf{J}}_{<t}, \phi\right]\right), \bar{\Psi}_{t_{0} \mathbf{r}^{N}}^{\min }=\Psi_{t_{0} \mathbf{r}^{N}}
\end{aligned}
$$

which implies that $\bar{\Psi}_{t \mathbf{r}^{N}}^{\min }\left[\overline{\mathbf{J}}_{<t}, \phi\right] \equiv \bar{\Psi}_{t \mathbf{r}^{N}}^{\min }\left[\overline{\mathbf{J}}_{<t}\right]$ is in fact independent of the external field entirely. Thus, it is, according the usual terminology of DFT, a universal functional (given an initial condition) satisfying the equation

$$
\begin{aligned}
\frac{\partial}{\partial t} \bar{\Psi}_{t \mathbf{r}^{N}}^{\min }[\overline{\mathbf{J}}]= & -\sum_{i} \nabla_{i} \cdot \frac{1}{\gamma} \mathbf{F}_{\mathbf{r}^{N}}^{\operatorname{tot}(i)}\left[0, \bar{\Psi}_{t}^{\min }\right] \bar{\Psi}_{t \mathbf{r}^{N}}[\overline{\mathbf{J}}]+ \\
& \sum_{i} \nabla_{i} \cdot\left(\frac{\mathbf{J}_{\mathbf{r}_{i}}\left[0, \bar{\Psi}_{t}^{\min }\right]-\overline{\mathbf{J}}_{t \mathbf{r}_{i}}}{\rho_{t \mathbf{r}_{i}}[\overline{\mathbf{J}}]} \bar{\Psi}_{t \mathbf{r}^{N}}[\overline{\mathbf{J}}]\right), \bar{\Psi}_{t_{0} \mathbf{r}^{N}}=\Psi_{t_{0} \mathbf{r}^{N}}
\end{aligned}
$$


As already noted, the minimization with respect to the current for all times gives

$$
\overline{\mathbf{J}}_{t \mathbf{r}}^{\min }=\mathbf{J}_{\mathbf{r}}\left[\phi, \bar{\Psi}_{t}^{\min }\right]
$$

which (using the definitions in Eqs. (8) and (11)) can be written as

$$
\overline{\mathbf{J}}_{t_{i}}^{\min }=-\frac{k_{B} T}{\gamma} \nabla \rho_{\mathbf{r}_{i}}[\overline{\mathbf{J}}]-\frac{1}{\gamma} \rho_{\mathbf{r}}[\overline{\mathbf{J}}]\left(\nabla \phi_{t \mathbf{r}}\right)+\left.\mathbf{K}_{t \mathbf{r}}[\overline{\mathbf{J}}]\right|_{\overline{\mathbf{J}}=\overline{\mathbf{J}}^{\min }}
$$

where the last term is

$$
\mathbf{K}_{t \mathbf{r}}[\overline{\mathbf{J}}]=-\frac{1}{\gamma} \int \sum_{i} \delta\left(\mathbf{r}-\mathbf{r}_{i}\right)\left(\left(\nabla_{i} U\left(\mathbf{r}^{N}\right)\right) \bar{\Psi}_{t \mathbf{r}^{N}}^{\min }[\overline{\mathbf{J}}]\right) d \mathbf{r}^{N}
$$

Although not indicated, everything also depends on the initial condition for the distribution, $\Psi_{t_{0} \mathbf{r}^{N}}$. Thus, we can summarize the evolution of the physical density with the following exact equations

$$
\begin{aligned}
\frac{\partial}{\partial t} \rho_{t \mathbf{r}}\left[\overline{\mathbf{J}}^{\min }\right] & =-\nabla \cdot \overline{\mathbf{J}}_{t \mathbf{r}}^{\min } \\
\overline{\mathbf{J}}_{t \mathbf{r}_{i}}^{\min } & =-\frac{k_{B} T}{\gamma} \nabla \rho_{t \mathbf{r}_{i}}\left[\overline{\mathbf{J}}^{\mathrm{min}}\right]-\frac{1}{\gamma} \rho_{t \mathbf{r}}\left[\overline{\mathbf{J}}^{\min }\right]\left(\nabla \phi_{t \mathbf{r}}\right)+\mathbf{K}_{t \mathbf{r}}\left[\overline{\mathbf{J}}^{\mathrm{min}}\right]
\end{aligned}
$$

For an ideal gas, $\mathbf{K}_{t \mathbf{r}}=0$ by definition and this is exact - not as a consequence of an ansatz but directly demonstrated from the microscopic formalism. In equilibrium, the distribution is constant so $\bar{\Psi}_{t_{\mathbf{r}}}^{\min }[\overline{\mathbf{J}}]=\Psi_{t_{0} \mathbf{r}^{N}}$ and so

$$
\mathbf{K}_{t \mathbf{r}}^{\text {equil }}[\overline{\mathbf{J}}]=-\frac{1}{\gamma} \int \sum_{i} \delta\left(\mathbf{r}-\mathbf{r}_{i}\right)\left(\left(\nabla_{i} U\left(\mathbf{r}^{N}\right)\right) \Psi_{t_{0} \mathbf{r}^{N}}^{\text {equil }}\right) d \mathbf{r}^{N}=-\frac{1}{\gamma} \nabla \frac{\delta F^{(\mathrm{ex})}[\rho]}{\delta \rho_{t \mathbf{r}}},
$$

where $F^{(\mathrm{ex})}[\rho]$ is the excess Helmholtz free energy functional of classical DFT. This is again an exact result (essentially, a consequence of the YBG hierarchy - see, e.g. Ref.[4]). Thus, a natural local-equilibrium assumption would be to continue to use this form for non-equilibrium systems resulting in

$$
\overline{\mathbf{J}}_{t \mathbf{r}_{i}}^{\min }=-\frac{k_{B} T}{\gamma} \nabla \rho_{t \mathbf{r}_{i}}\left[\overline{\mathbf{J}}^{\mathrm{min}}\right]-\frac{1}{\gamma} \rho_{t \mathbf{r}}\left[\overline{\mathbf{J}}^{\mathrm{min}}\right]\left(\nabla \phi_{t \mathbf{r}}\right)-\frac{1}{\gamma} \nabla\left(\frac{\delta F^{(\mathrm{ex})}[\rho]}{\delta \rho_{t \mathbf{r}}}\right)_{\rho_{t \mathbf{r}}\left[\overline{\mathbf{J}}^{\mathrm{min}}\right]}
$$

which, when inserted into the continuity equation, gives a closed dynamics for the density usually referred to as Dynamic Density Functional Theory (DDFT).

The investigation of deviations between the true dynamics and the dynamics predicted by DDFT (adiabatic dynamics in the sense of a dynamics of quasi-static changes) has been 
pursued by Schmidt and coworkers over the past years, see e.g. [19, 20]. Even without knowing the explicit form of the equilibrium free energy functional the adiabatic dynamics can be determined by simulation [19] and thus also the nonadiabatic differences to the true dynamics. In terms of the ansatz in Eq. (30), these differences are contained in the excess part $P_{t}^{\text {ex }}$ of the functional $P_{t}[\overline{\mathbf{J}}]$ which is defined as follows:

$$
P_{t}[\overline{\mathbf{J}}]=P_{t}^{(\mathrm{id})}[\overline{\mathbf{J}}]+P_{t}^{(\mathrm{ex})}[\overline{\mathbf{J}}] \quad \text { with } \quad P_{t}^{\mathrm{id}}[\overline{\mathbf{J}}]=\frac{\gamma}{2} \int \frac{\overline{\mathbf{J}}_{t \mathbf{r}}^{2}}{\rho_{t \mathbf{r}}} d \mathbf{r}
$$

The relation to the functional $K_{t \mathbf{r}}$ defined above is given by

$$
K_{t \mathbf{r}}[\overline{\mathbf{J}}]=\frac{\delta P_{t}^{\operatorname{ex}}[\overline{\mathbf{J}}]}{\delta \overline{\mathbf{J}}}+\frac{1}{\gamma} \nabla\left(\frac{\delta F^{(\mathrm{ex})}[\rho]}{\delta \rho_{t \mathbf{r}}}\right)_{\rho_{t \mathbf{r}}[\overline{\mathbf{J}}]}
$$

Note that we have restricted the functional dependence of the $P_{t}$ functional to the current only, in line with our modified formulation of PFT.

\section{CONCLUSIONS}

We have attempted to clarify the construction of Power Functional Theory for Brownian dynamics. The theory begins with a simple variational principle that reproduces the exact statistical description of the system based on a generating function ultimately related to the Rayleigh dissipation function. The minimization is broken into two steps - first, minimization under constraints of constant density and current, followed by minimization over those fields. The first minimization results in a functional of the current and density fields called the power functional. This is meant to be analogous to the Helmholtz functional of cDFT and to play a similar role as a starting point for physically motivated approximations to the dynamics of the full $N$-body system. Our analysis has identified several problematic points. There is some ambiguity in the development of SB as to whether the power functional is actually functional of both density and current fields or only of the current. A dependence on both seems to lead to unphysical results so that it should probably be considered as the result of a constrained minimization at fixed current. Independent of this, the resulting expressions do not have the structure described by SB which in turn has been used in practical applications of the theory lateron, and thus the status of those is uncertain and the relation to the underlying power functional is unclear. 
Having identified the source of the problems in the original development, we have proposed modifications which appear to avoid them. The result is in some ways very similar to that discussed by SB but with one critical difference: in our development it is possible to carry through the first constrained minimization exactly resulting in the exact power functional. This turns out to be a rather trivial quadratic function that simply forces the current to take on its exact expression. The theory therefore seems to provide no real advantage over, for example, simply introducing approximations directly into the continuity equation.

More optimistically, we noted that our development does result in an interesting reformulation of the dynamics whereby a "universal" functional of the current is defined (i.e. one that is independent of the external field) and that can be used to formulate the dynamics governing the local density. Dropping unnecessary notation, the resulting theory has the form

$$
\begin{aligned}
\frac{\partial}{\partial t} \rho_{t \mathbf{r}} & =-\nabla \cdot \mathbf{J}_{t \mathbf{r}} \\
\mathbf{J}_{t \mathbf{r}} & =-\frac{k_{B} T}{\gamma} \nabla \rho_{t \mathbf{r}}-\frac{1}{\gamma} \rho_{t \mathbf{r}}\left(\nabla \phi_{t \mathbf{r}}\right)+\mathbf{K}_{t \mathbf{r}}[\mathbf{J}]
\end{aligned}
$$

with the non-ideal - or excess - contribution to the current given by

$$
\mathbf{K}_{t \mathbf{r}}[\mathbf{J}]=-\frac{1}{\gamma} \int \sum_{i} \delta\left(\mathbf{r}-\mathbf{r}_{i}\right) \Psi_{t \mathbf{r}^{N}}[\mathbf{J}] \nabla_{i} U\left(\mathbf{r}^{N}\right) d \mathbf{r}^{N}
$$

and finally the "universal" distribution determined by $\left(\Psi_{\mathbf{r}^{N}}^{(\mathrm{ini})}\right.$ is the distribution specifying initial conditions)

$$
\begin{aligned}
\frac{\partial}{\partial t} \Psi_{t \mathbf{r}^{N}}[\mathbf{J}]= & -\sum_{i} \nabla_{i} \cdot \frac{1}{\gamma} \mathbf{F}_{\mathbf{r}^{N}}^{\text {tot }(i)}[0, \Psi] \Psi_{t \mathbf{r}^{N}}[\mathbf{J}]+\sum_{i} \nabla_{i} \cdot\left(\frac{\mathbf{J}_{\mathbf{r}_{i}}[0, \Psi]-\mathbf{J}_{t \mathbf{r}_{i}}}{\rho_{t \mathbf{r}_{i}}} \Psi_{t \mathbf{r}^{N}}[\mathbf{J}]\right) \\
& \text { with } \Psi_{t_{0} \mathbf{r}^{N}}=\Psi_{\mathbf{r}^{N}}^{(\mathrm{ini})}
\end{aligned}
$$

This is similar to the structure of classical DFT in which one has a universal functional of the density, the so-called Helmholtz functional $F[\rho]$, and from this one constructs simple one-body equations for the local density given an external field. Here $\mathbf{K}_{t \mathbf{r}}[\mathbf{J}]$ plays the role of the Helmholtz functional and it shares another characteristic as well: its determination depends on solving an $N$-body problem that is as complicated as simply solving the original Fokker-Planck equation (or, in the case of cDFT calculating the original partition function) that defined the starting point. Whether there is any advantage to such a reformulation of the original problem remains to be seen. 


\section{ACKNOWLEDGMENTS}

The work of JFL was supported by the European Space Agency (ESA) and the Belgian Federal Science Policy Office (BELSPO) in the framework of the PRODEX Programme, contract number ESA AO-2004-070. MO acknowlegdes funding by the German Research Foundation (DFG) through grant OE 285/6-1 within the priority program SPP2171.

\section{DATA AVAILABILITY}

Data sharing is not applicable to this article as no new data were created or analyzed in this study.

\section{Appendix A: Derivation of the form of the generating functional}

SB begin by defining (their Eq.(10))

$$
\widehat{R}_{t \mathbf{r}^{N}}\left[\widetilde{\mathbf{v}}^{N}\right]=\sum_{i}\left(\frac{\gamma}{2} \widetilde{\mathbf{v}}_{t \mathbf{r}^{N}}^{(i)}-\mathbf{F}_{t \mathbf{r}^{N}}^{\mathrm{tot}(i)}\right) \cdot \widetilde{\mathbf{v}}_{t \mathbf{r}^{N}}^{(i)}+\dot{\phi}_{t \mathbf{r}^{N}}
$$

with (SB Eq.(5))

$$
\mathbf{F}_{t \mathbf{r}^{N}}^{\mathrm{tot}(i)}\left[\phi_{t}, \Psi\right]=-\nabla_{i} U_{\mathbf{r}^{N}}-\nabla_{i} \phi_{t \mathbf{r}^{N}}-k_{B} T \nabla_{i} \ln \Psi_{t \mathbf{r}}
$$

and (SB Eq.(17))

$$
\gamma \widetilde{\mathbf{v}}_{t \mathbf{r}^{N}}^{(i)} \equiv \gamma \mathbf{v}_{t \mathbf{r}^{N}}^{(i)}\left[\phi_{t}, \widetilde{\Psi}\right]=\mathbf{F}_{t \mathbf{r}^{N}}^{\mathrm{tot}(i)}\left[\phi_{t}, \widetilde{\Psi}\right]
$$

in terms of which the Fokker-Planck equation becomes

$$
\frac{\partial}{\partial t} \Psi_{t \mathbf{r}^{N}}=-\frac{1}{\gamma} \sum_{i=1}^{N} \nabla_{i} \cdot\left(\Psi_{t \mathbf{r}} \mathbf{F}_{t \mathbf{r}^{N}}^{\mathrm{tot}(i)}\left[\phi_{t}, \Psi\right]\right)
$$

The functional $\widehat{R}_{t^{N}}$ can be written as

$$
\begin{aligned}
\widehat{R}_{t \mathbf{r}^{N}}\left[\widetilde{\mathbf{v}}^{N}\right] & =\frac{\gamma}{2} \sum_{i}\left(\widetilde{\mathbf{v}}_{t \mathbf{r}^{N}}^{(i)}-\frac{1}{\gamma} \mathbf{F}_{t \mathbf{r}^{N}}^{\mathrm{tot}(i)}\right)^{2}-\frac{1}{2 \gamma} \sum_{i}\left(\mathbf{F}_{t \mathbf{r}^{N}}^{\mathrm{tot}(i)}\right)^{2}+\dot{\phi}_{t \mathbf{r}^{N}} \\
& =\frac{\left(k_{B} T\right)^{2} \gamma}{2} \sum_{i}\left(\nabla_{i} \ln \frac{\widetilde{\Psi}_{\mathbf{r}}}{\Psi_{\mathbf{r}}}\right)^{2}-\frac{1}{2 \gamma} \sum_{i}\left(\mathbf{F}_{t \mathbf{r}^{N}}^{\mathrm{tot}(i)}\right)^{2}+\dot{\phi}_{t \mathbf{r}^{N}}
\end{aligned}
$$


The SB generating functional is (SB Eq.(11))

$$
\begin{aligned}
R\left[\widetilde{\Psi} ; \Psi_{t}\right] & =\int \Psi_{t \mathbf{r}^{N}} \widehat{R}_{t \mathbf{r}^{N}} d \mathbf{r}^{N} \\
& =\frac{\left(k_{B} T\right)^{2} \gamma}{2} \int \sum_{i}\left(\nabla_{i} \ln \frac{\widetilde{\Psi}_{\mathbf{r}}}{\Psi_{\mathbf{r}}}\right)^{2} \Psi_{t \mathbf{r}^{N}} d \mathbf{r}^{N} \\
& -\frac{1}{2 \gamma} \int \Psi_{t \mathbf{r}^{N}} \sum_{i}\left(\mathbf{F}_{\mathbf{r}^{N}}^{\operatorname{tot}(i)}\left[\Psi_{t}, \phi_{t}\right]\right)^{2} d \mathbf{r}^{N} \\
& +\int \Psi_{t \mathbf{r}^{N}} \dot{\phi}_{t \mathbf{r}^{N}} d \mathbf{r}^{N}
\end{aligned}
$$

The second term is

$$
\begin{aligned}
-\frac{1}{2 \gamma} \int \Psi_{t} \sum_{i}\left(\mathbf{F}_{\mathbf{r}^{N}}^{\mathrm{tot}(i)}\left[\Psi_{t}, \phi_{t}\right]\right)^{2} d \mathbf{r}^{N} & =\frac{1}{2 \gamma} \int \sum_{i}\left(\nabla_{i}\left(U_{\mathbf{r}^{N}}+\phi_{t \mathbf{r}^{N}}+k_{B} T \ln \Psi_{t \mathbf{r}^{N}}\right)\right) \cdot \Psi_{t \mathbf{r}^{N}} \mathbf{F}_{\mathbf{r}^{N}}^{\mathrm{tot}(i)}\left[\Psi_{t}, \phi_{t}\right] d \mathbf{r}^{N} \\
& =-\frac{1}{2 \gamma} \int\left(U_{\mathbf{r}^{N}}+\phi_{t \mathbf{r}^{N}}+k_{B} T \ln \Psi_{t \mathbf{r}^{N}}\right) \sum_{i} \nabla_{i} \cdot \Psi_{t \mathbf{r}^{N}} \mathbf{F}_{\mathbf{r}^{N}}^{\mathrm{tot}(i)}\left[\Psi_{t}, \phi_{t}\right] d \mathbf{r}^{N}
\end{aligned}
$$

where the second line follows from the divergence theorem and surface terms are ignored.

Substituting for the divergence using the Fokker-Planck equation,

$$
\begin{aligned}
-\frac{1}{2 \gamma} \int \Psi_{t} \sum_{i}\left(\mathbf{F}_{t \mathbf{r}^{N}}^{\mathrm{tot}(i)}\right)^{2} d \mathbf{r}^{N} & =-\frac{1}{2 \gamma} \int\left(U_{\mathbf{r}^{N}}+\phi_{t \mathbf{r}^{N}}+k_{B} T \ln \Psi_{t \mathbf{r}^{N}}\right)\left(-\gamma \frac{\partial}{\partial t} \Psi_{t \mathbf{r}^{N}}\right) d \mathbf{r}^{N} \\
& =\frac{1}{2} \frac{\partial}{\partial t} \int\left(U_{\mathbf{r}^{N}}+\phi_{t \mathbf{r}^{N}}+k_{B} T \ln \Psi_{t \mathbf{r}^{N}}\right) \Psi_{t \mathbf{r}^{N}} d \mathbf{r}^{N}-\frac{1}{2} \int\left(\frac{\partial}{\partial t} \phi_{t \mathbf{r}^{N}}\right) \Psi_{t \mathbf{r}^{N}} d \mathbf{r}^{N} \\
& =\frac{1}{2} \frac{\partial}{\partial t} \Lambda\left[\phi_{t}, \Psi_{t}\right]-\frac{1}{2} \int\left(\frac{\partial}{\partial t} \phi_{t \mathbf{r}^{N}}\right) \Psi_{t \mathbf{r}^{N}} d \mathbf{r}^{N}
\end{aligned}
$$

Putting everything together gives the form used in the main text,

$$
R\left[\widetilde{\Psi} ; \Psi_{t}\right]=\frac{\left(k_{B} T\right)^{2} \gamma}{2} \int \sum_{i}\left(\nabla_{i} \ln \frac{\widetilde{\Psi}_{t \mathbf{r}^{N}}}{\Psi_{t \mathbf{r}^{N}}}\right)^{2} \Psi_{t \mathbf{r}^{N}} d \mathbf{r}^{N}+\frac{1}{2} \frac{\partial}{\partial t} \Lambda\left[\phi_{t}, \Psi_{t}\right]+\frac{1}{2} \int \Psi_{t \mathbf{r}^{N}} \dot{\phi}_{t \mathbf{r}^{N}} d \mathbf{r}^{N}
$$

In order to arrive at Eq.(27) of the main text, we need to evaluate

$$
\overline{\mathcal{R}}_{t}\left[\bar{\rho}, \overline{\mathbf{J}} ; \phi_{t}, \dot{\phi}_{t}\right]=R\left[\widetilde{\Psi}^{\min }[\bar{\rho}, \overline{\mathbf{J}}] ; \phi_{t}, \dot{\phi}_{t}, \bar{\Psi}_{t}[\bar{\rho}, \overline{\mathbf{J}}]\right]
$$

or, using Eq.(A5) and dropping all unnecessary functional arguments for the sake of clarity,

$$
\begin{aligned}
\overline{\mathcal{R}}_{t}\left[\bar{\rho}, \overline{\mathbf{J}} ; \phi_{t}, \dot{\phi}_{t}\right]= & \frac{\left(k_{B} T\right)^{2} \gamma}{2} \int \sum_{i}\left(\nabla_{i} \ln \frac{\widetilde{\Psi}_{t \mathbf{r}}^{\min }}{\bar{\Psi}_{t \mathbf{r}}}\right)^{2} \bar{\Psi}_{t \mathbf{r}^{N}} d \mathbf{r}^{N} \\
& -\frac{1}{2 \gamma} \int \bar{\Psi}_{t \mathbf{r}^{N}} \sum_{i}\left(\mathbf{F}_{\mathbf{r}^{N}}^{\mathrm{tot}(i)}\left[\bar{\Psi}_{t}, \phi_{t}\right]\right)^{2} d \mathbf{r}^{N}+\int \bar{\Psi}_{t \mathbf{r}^{N}} \dot{\phi}_{t \mathbf{r}^{N}} d \mathbf{r}^{N}
\end{aligned}
$$


Now, given the linear dependence of $\mathbf{F}_{\mathbf{r}^{N}}^{\mathrm{tot}(i)}$ on the field,

$$
\mathbf{F}_{\mathbf{r}^{N}}^{\mathrm{tot}(i)}[\phi, \Psi]=\mathbf{F}_{\mathbf{r}^{N}}^{\mathrm{tot}(i)}[0, \Psi]-\nabla_{i} \phi_{\mathbf{r}^{N}}
$$

one has that

$$
\begin{aligned}
-\frac{1}{2 \gamma} \int \bar{\Psi}_{\mathbf{r}^{N}} \sum_{i}\left(\mathbf{F}_{\mathbf{r}^{N}}^{\mathrm{tot}(i)}[\phi, \bar{\Psi}]\right)^{2} d \mathbf{r}^{N} & =-\frac{1}{2 \gamma} \int \bar{\Psi}_{\mathbf{r}^{N}} \sum_{i}\left(\mathbf{F}_{\mathbf{r}^{N}}^{\mathrm{tot}(i)}[0, \bar{\Psi}]\right)^{2} d \mathbf{r}^{N} \\
& +\frac{1}{\gamma} \int \bar{\Psi}_{\mathbf{r}^{N}} \sum_{i}\left(\mathbf{F}_{\mathbf{r}^{N}}^{\mathrm{tot}(i)}[0, \bar{\Psi}] \cdot \nabla_{i} \phi_{\mathbf{r}_{i}}\right) d \mathbf{r}^{N} \\
& -\frac{1}{2 \gamma} \int \bar{\Psi}_{t^{N}} \sum_{i}\left(\nabla_{i} \phi_{\mathbf{r}_{i}}\right)^{2} d \mathbf{r}^{N}
\end{aligned}
$$

The second term is

$$
\begin{aligned}
\frac{1}{\gamma} \int \bar{\Psi}_{\mathbf{r}^{N}} \sum_{i}\left(\mathbf{F}_{\mathbf{r}^{N}}^{\mathrm{tot}(i)}\left[0, \bar{\Psi} \cdot \nabla_{i} \phi_{\mathbf{r}_{i}}\right) d \mathbf{r}^{N}\right. & =\int \bar{\Psi}_{\mathbf{r}^{N}} \sum_{i}\left(\frac{1}{\gamma} \mathbf{F}_{\mathbf{r}^{N}}^{\mathrm{tot}(i)}[0, \bar{\Psi}]\left\{\int\left(\nabla \phi_{\mathbf{r}}\right) \delta\left(\mathbf{r}-\mathbf{r}_{i}\right) d \mathbf{r}\right\}\right) d \mathbf{r}^{N} \\
& =\int\left(\nabla \phi_{\mathbf{r}}\right) \cdot\left\{\int \delta\left(\mathbf{r}-\mathbf{r}_{i}\right) \bar{\Psi}_{\mathbf{r}^{N}} \sum_{i}\left(\frac{1}{\gamma} \mathbf{F}_{\mathbf{r}^{N}}^{\mathrm{tot}(i)}[0, \bar{\Psi}]\right) d \mathbf{r}^{N}\right\} d \mathbf{r} \\
& =\int\left(\nabla \phi_{\mathbf{r}}\right) \cdot \mathbf{J}[0, \bar{\Psi}] d \mathbf{r}
\end{aligned}
$$

Thus

$$
\begin{aligned}
\overline{\mathcal{R}}_{t}\left[\bar{\rho}, \overline{\mathbf{J}} ; \phi_{t}, \dot{\phi}_{t}\right] & =\frac{\left(k_{B} T\right)^{2} \gamma}{2} \int \sum_{i}\left(\nabla_{i} \ln \frac{\widetilde{\Psi}_{t \mathbf{r}}^{\min }}{\bar{\Psi}_{t \mathbf{r}}}\right)^{2} \bar{\Psi}_{t \mathbf{r}^{N}} d \mathbf{r}^{N}-\frac{1}{2 \gamma} \int \bar{\Psi}_{t \mathbf{r}^{N}} \sum_{i}\left(\mathbf{F}_{\mathbf{r}^{N}}^{\mathrm{tot}(i)}\left[0, \bar{\Psi}_{t}\right]\right)^{2} d \mathbf{r}^{N} \\
& +\int\left(\nabla \phi_{t \mathbf{r}}\right) \cdot \mathbf{J}_{\mathbf{r}}\left[0, \bar{\Psi}_{t}\right] d \mathbf{r}-\frac{1}{2 \gamma} \int\left(\nabla \phi_{t \mathbf{r}}\right)^{2} \rho_{\mathbf{r}}\left[\bar{\Psi}_{t}\right] d \mathbf{r}^{N}+\int \rho_{\mathbf{r}}\left[\bar{\Psi}_{t}\right] \dot{\phi}_{t \mathbf{r}^{N}} d \mathbf{r}^{N}
\end{aligned}
$$

which is Eq. (27) in the main text.

\section{Appendix B: Minimizing with independent density and current}

To be of use, models must be developed for the functionals to be minimized. SB propose to write the functional $W_{t}^{(S B)}$ as

$$
W_{t}^{(S B)}\left[\bar{\rho}, \overline{\mathbf{J}} ; \phi_{t}, \Psi_{t}\right]=P^{(\mathrm{ex})}\left[\bar{\rho}, \overline{\mathbf{J}} ; \phi_{t}, \Psi_{t}\right]+\int\left(\frac{\overline{\mathbf{J}}_{\mathbf{r}^{\prime}}^{2}}{2 \gamma \bar{\rho}_{\mathbf{r}^{\prime}}}+\overline{\mathbf{J}}_{\mathbf{r}^{\prime}} \cdot \nabla \frac{\delta F[\bar{\rho}]}{\delta \bar{\rho}_{\mathbf{r}^{\prime}}}\right) d \mathbf{r}^{\prime}
$$


where $P^{(\mathrm{ex})}$ is the excess "dissipated power functional", the quadratic term in $\overline{\mathbf{J}}$ is the "ideal dissipated power functional" and the third term is the "adiabatic" (i.e. local equilibrium) contribution which involves the DFT free energy functional $F\left[\bar{\rho}_{t}\right]$ (see Eqs. (30) and (62)). The Euler-Lagrange equations then become

$$
\begin{array}{r}
\frac{\delta}{\delta \bar{\rho}_{\mathbf{r}}} P^{(\mathrm{ex})}\left[\bar{\rho}, \overline{\mathbf{J}} ; \phi_{t}, \Psi_{t}\right]-\frac{\overline{\mathbf{J}}_{\mathbf{r}}^{2}}{2 \gamma \bar{\rho}_{\mathbf{r}}^{2}}-\int\left(\nabla^{\prime} \cdot \overline{\mathbf{J}}_{\mathbf{r}^{\prime}}\right) \frac{\delta^{2} F[\bar{\rho}]}{\delta \bar{\rho}_{\mathbf{r}} \delta \bar{\rho}_{\mathbf{r}^{\prime}}} d \mathbf{r}^{\prime}=0 \\
\frac{\delta}{\delta \overline{\mathbf{J}}_{\mathbf{r}}} P^{(\mathrm{ex})}\left[\bar{\rho}, \overline{\mathbf{J}} ; \phi_{t}, \Psi_{t}\right]+\frac{\overline{\mathbf{J}}_{\mathbf{r}}}{\gamma \bar{\rho}_{\mathbf{r}}}+\nabla \frac{\delta F[\bar{\rho}]}{\delta \bar{\rho}_{\mathbf{r}}}+\nabla \phi_{t \mathbf{r}}=0
\end{array}
$$

Separating the equilibrium free energy functional into its ideal and excess parts $\left(F=F^{(\mathrm{id})}+\right.$

$F^{(\text {ex })}$ with $F^{(\text {id })}[\rho]=k_{B} T \int d \mathbf{r} \rho(\mathbf{r})\left(\ln \left(\rho(\mathbf{r}) \lambda^{3}\right)-1\right)$ where $\lambda$ is the thermal de-Broglie length $)$ and rearranging gives

$$
\begin{array}{r}
\bar{\rho}_{\mathbf{r}}^{2} \frac{\delta}{\delta \bar{\rho}_{\mathbf{r}}} P^{(\mathrm{ex})}\left[\bar{\rho}, \overline{\mathbf{J}} ; \phi_{t}, \Psi_{t}\right]-\frac{1}{2 \gamma} \overline{\mathbf{J}}_{\mathbf{r}}^{2}-\bar{\rho}_{\mathbf{r}}\left(\nabla \cdot \overline{\mathbf{J}}_{\mathbf{r}}\right)-\bar{\rho}_{\mathbf{r}}^{2} \int\left(\nabla^{\prime} \cdot \overline{\mathbf{J}}_{\mathbf{r}^{\prime}}\right) \frac{\delta^{2} F^{(\mathrm{ex})}[\bar{\rho}]}{\delta \bar{\rho}_{\mathbf{r}} \delta \bar{\rho}_{\mathbf{r}^{\prime}}} d \mathbf{r}^{\prime}=0 \\
\bar{\rho}_{\mathbf{r}} \frac{\delta}{\delta \overline{\mathbf{J}}_{\mathbf{r}}} P^{(\mathrm{ex})}\left[\bar{\rho}, \overline{\mathbf{J}} ; \phi_{t}, \Psi_{t}\right]+\frac{\overline{\mathbf{J}}_{\mathbf{r}}}{\gamma}+\nabla \bar{\rho}_{\mathbf{r}}+\bar{\rho}_{\mathbf{r}} \nabla \frac{\delta F^{(\mathrm{ex})}[\bar{\rho}]}{\delta \bar{\rho}_{\mathbf{r}}}+\bar{\rho}_{\mathbf{r}} \nabla \phi_{t \mathbf{r}}=0
\end{array}
$$

This completes as much of the general framework as laid out by SB and needed here and now we turn to applications to specific systems.

\section{Equilibrium}

In equilibrium, there is no time dependence and the current vanishes. We assume that the contribution from $P^{(\mathrm{ex})}$ also vanishes so that the only non-trivial equation is the second (coming from the variation of the current) which can be written as

$$
\bar{\rho}_{\mathbf{r}} \nabla \frac{\delta}{\delta \bar{\rho}_{\mathbf{r}}}\left(F[\bar{\rho}]+\int \bar{\rho}_{\mathbf{r}^{\prime}} \phi_{\mathbf{r}^{\prime}} d \mathbf{r}^{\prime}\right)=0
$$

and which has the usual equilibrium DFT solution

$$
F[\bar{\rho}]+\int \bar{\rho}_{\mathbf{r}^{\prime}} \phi_{\mathbf{r}^{\prime}} d \mathbf{r}^{\prime}=\text { const. }
$$

In this sense, the proposed ansatz for $W$ reproduces equilibrium cDFT. 


\section{Adiabatic approximation}

By the definition of SB, "adiabatic" means that one ignores the excess dissipated power functional $\left(P^{(\mathrm{ex})}=0\right)$ giving

$$
\begin{aligned}
0 & =\frac{1}{2 \gamma} \overline{\mathbf{J}}_{\mathbf{r}}^{2}+\bar{\rho}_{\mathbf{r}}\left(\nabla \cdot \overline{\mathbf{J}}_{\mathbf{r}}\right)+\bar{\rho}_{\mathbf{r}}^{2} \int\left(\nabla^{\prime} \cdot \overline{\mathbf{J}}_{\mathbf{r}^{\prime}} \frac{\delta^{2} F^{(\mathrm{ex})}[\bar{\rho}]}{\delta \bar{\rho}_{\mathbf{r}} \delta \bar{\rho}_{\mathbf{r}^{\prime}}}\right) d \mathbf{r}^{\prime} \\
\frac{1}{\gamma} \overline{\mathbf{J}}_{\mathbf{r}} & =-\nabla \bar{\rho}_{\mathbf{r}}-\bar{\rho}_{\mathbf{r}} \nabla \frac{\delta F^{(\mathrm{ex})}[\bar{\rho}]}{\delta \bar{\rho}_{\mathbf{r}}}-\bar{\rho}_{\mathbf{r}} \nabla \phi_{t \mathbf{r}}
\end{aligned}
$$

The second equation gives the current in terms of the density and external field and if this is inserted into the continuity equation, the resulting equation of motion can be written as

$$
\frac{\partial}{\partial t} \bar{\rho}_{\mathbf{r}}=+\nabla \cdot \bar{\rho}_{\mathbf{r}} \nabla \frac{\delta}{\delta \bar{\rho}_{\mathbf{r}}}\left(F[\bar{\rho}]+\int \bar{\rho}_{\mathbf{r}^{\prime}} \phi_{t \mathbf{r}^{\prime}} d \mathbf{r}^{\prime}\right)
$$

which looks like the standard DDFT equation of motion. However, as emphasized above, there is no reason to suppose that the density and current satisfy the continuity equation and, indeed, as the notation makes clear, $\bar{\rho}_{\mathbf{r}}$ is a time-independent test-field so that this equation makes little sense. Rather, the density should be determined from the first of Eqs.(B6), which is purely local in time. Denoting the solution of the Euler-Lagrange equation as

$$
\bar{\rho}_{\mathbf{r}}^{*}\left[\phi_{t}\right], \overline{\mathbf{J}}_{\mathbf{r}}^{*}\left[\phi_{t}\right]
$$

since the only time-dependence in Eq. (B6 $)$ occurs via the potential, and substituting into the continuity equation gives

$$
\int \frac{\delta \bar{\rho}_{\mathbf{r}}^{*}\left[\phi_{t}\right]}{\delta \phi_{t \mathbf{r}^{\prime}}} \frac{\partial \phi_{t \mathbf{r}^{\prime}}}{\partial t} d \mathbf{r}^{\prime}=-\nabla \cdot \overline{\mathbf{J}}_{\mathbf{r}}^{*}\left[\phi_{t}\right]
$$

which can only be satisfied for particular fields. In particular, it is clear that in the case of an external field that does not depend on time, one could start with a density which is not the equilibrium density, so that presumably $\bar{\rho}_{\mathbf{r}}^{*}$ will relax to its equilibrium value. In such a case, the left-hand side vanishes, but $\overline{\mathbf{J}}_{\mathbf{r}}^{*}$ will be nonzero, and indeed nontrivial, so that the right hand side does not vanish, thus showing that such a solution cannot be consistent with the continuity equation. To understand this result better, we turn to the final special case, the ideal gas. 


\section{Ideal gas in the adiabatic approximation}

For the ideal gas, the excess part of the free energy functional vanishes leaving

$$
\begin{aligned}
\frac{1}{2 \gamma} \overline{\mathbf{J}}_{\mathbf{r}}^{2}+\bar{\rho}_{\mathbf{r}}\left(\nabla \cdot \overline{\mathbf{J}}_{\mathbf{r}}\right) & =0 \\
\frac{1}{\gamma} \overline{\mathbf{J}}_{\mathbf{r}} & =-\nabla \bar{\rho}_{\mathbf{r}}-\bar{\rho}_{\mathbf{r}} \nabla \beta \phi_{t \mathbf{r}} \quad\left(\beta=1 /\left(k_{B} T\right)\right) .
\end{aligned}
$$

The equation for the current is in fact the exact result which can be derived directly from the Fokker-Planck equation. It can be used to eliminate the current from the first equation giving

$$
\bar{\rho}_{\mathbf{r}}\left(\nabla^{2} \bar{\rho}_{\mathbf{r}}\right)-\frac{1}{2}\left(\nabla \bar{\rho}_{\mathbf{r}}\right)^{2}+\bar{\rho}_{\mathbf{r}}^{2}\left(\nabla^{2} \beta \phi_{t \mathbf{r}}-\frac{1}{2}\left(\nabla \beta \phi_{t \mathbf{r}}\right)^{2}\right)=0
$$

which is satisfied by the adiabatic solution

$$
\bar{\rho}_{\mathbf{r}}^{*}\left[\phi_{t}\right]=A e^{-\beta \phi_{t \mathbf{r}}}
$$

for some constant $A$. However, this gives vanishing current and when substituted into the continuity equation gives

$$
\frac{\partial}{\partial t} \phi_{t \mathbf{r}}=0
$$

indicating that the minimizers $\bar{\rho}_{\mathbf{r}}^{*}$ and $\overline{\mathbf{J}}_{\mathbf{r}}^{*}$ are only consistent with the continuity equation in the particular case of a time-independent field.

[1] R. Evans, "The nature of the liquid-vapour interface and other topics in the statistical mechanics of non-uniform, classical fluids," Adv. Phys. 28, 143 (1979).

[2] James F. Lutsko, "Recent developments in classical density functional theory," Adv. Chem. Phys. 144, 1 (2010).

[3] Umberto Marini Bettolo Marconi and Pedro Tarazona, "Dynamic density functional theory of fluids," The Journal of Chemical Physics 110, 8032-8044 (1999).

[4] A. J. Archer and R. Evans, "Dynamical density functional theory and its application to spinodal decomposition," The Journal of Chemical Physics 121, 4246-4254 (2004).

[5] Benjamin D. Goddard, Andreas Nold, Nikos Savva, Grigorios A. Pavliotis, and Serafim Kalliadasis, "General dynamical density functional theory for classical fluids," Phys. Rev. Lett. 109, 120603 (2012). 
[6] Matthias Schmidt and Joseph M. Brader, "Power functional theory for brownian dynamics," The Journal of Chemical Physics 138, 214101 (2013).

[7] Matthias Schmidt, "Quantum power functional theory for many-body dynamics," The Journal of Chemical Physics 143, 174108 (2015).

[8] Matthias Schmidt, "Power functional theory for newtonian many-body dynamics," The Journal of Chemical Physics 148, 044502 (2018).

[9] Philip Krinninger and Matthias Schmidt, "Power functional theory for active brownian particles: General formulation and power sum rules," The Journal of Chemical Physics 150, $074112(2019)$.

[10] Thomas Schindler and Matthias Schmidt, "Dynamic pair correlations and superadiabatic forces in a dense brownian liquid," The Journal of Chemical Physics 145, 064506 (2016).

[11] Daniel de las Heras and Matthias Schmidt, "Flow and structure in nonequilibrium brownian many-body systems," Phys. Rev. Lett. 125, 018001 (2020).

[12] Michael te Vrugt, Hartmut Löwen, and Raphael Wittkowski, "Classical dynamical density functional theory: from fundamentals to applications," Advances in Physics 69, 121-247 (2020).

[13] Sophie Hermann, Philip Krinninger, Daniel de las Heras, and Matthias Schmidt, "Phase coexistence of active brownian particles," Phys. Rev. E 100, 052604 (2019).

[14] Sophie Hermann, Daniel de las Heras, and Matthias Schmidt, "Non-negative interfacial tension in phase-separated active brownian particles," Phys. Rev. Lett. 123, 268002 (2019).

[15] Sophie Hermann, Daniel de las Heras, and Matthias Schmidt, "Phase separation of active brownian particles in two dimensions: Anything for a quiet life," Molecular Physics , e1902585 (2021).

[16] BD Goddard, GA Pavliotis, and S Kalliadasis, "The overdamped limit of dynamic density functional theory: Rigorous results," Multiscale Modeling \& Simulation 10, 633-663 (2012).

[17] C. W. Gardiner, Handbook of Stochastic Methods, 3ed. (Springer, Berlin, 2004).

[18] N. David Mermin, "Thermal properties of the inhomogeneous electron gas," Phys. Rev. 137, A1441-A1443 (1965).

[19] Andrea Fortini, Daniel de las Heras, Joseph M. Brader, and Matthias Schmidt, "Superadiabatic forces in brownian many-body dynamics," Phys. Rev. Lett. 113, 167801 (2014). 
[20] Nico C. X. Stuhlmüller, Tobias Eckert, Daniel de las Heras, and Matthias Schmidt, "Structural nonequilibrium forces in driven colloidal systems," Phys. Rev. Lett. 121, 098002 (2018). 